\title{
One-particle irreducible functional approach: A route to diagrammatic extensions of the dynamical mean-field theory
}

\author{
G. Rohringer, ${ }^{1}$ A. Toschi, ${ }^{1}$ H. Hafermann, ${ }^{2}$ K. Held, ${ }^{1}$ V. I. Anisimov, ${ }^{3,4}$ and A. A. Katanin ${ }^{3,4}$ \\ ${ }^{1}$ Institute for Solid State Physics, Vienna University of Technology, 1040 Vienna, Austria \\ ${ }^{2}$ Centre de Physique Théorique, École Polytechnique, CNRS, 91128 Palaiseau Cedex, France \\ ${ }^{3}$ Institute of Metal Physics, 620990, Ekaterinburg, Russia \\ ${ }^{4}$ Ural Federal University, 620002, Ekaterinburg, Russia
}

(Received 2 April 2013; revised manuscript received 21 August 2013; published 9 September 2013)

\begin{abstract}
We present an approach which is based on the one-particle irreducible (1PI) generating functional formalism and includes electronic correlations on all length scales beyond the local correlations of dynamical mean-field theory (DMFT). This formalism allows us to unify aspects of the dynamical vertex approximation (DГA) and the dual fermion (DF) scheme, yielding a consistent formulation of nonlocal correlations at the one- and two-particle level beyond DMFT within the functional integral formalism. In particular, the considered approach includes one-particle reducible contributions from the three- and more-particle vertices in the dual fermion approach, as well as some diagrams not included in the ladder version of DГА. To demonstrate the applicability and physical content of the 1PI approach, we compare the diagrammatics of 1PI, DF, and DГA, as well as the numerical results of these approaches for the half-filled Hubbard model in two dimensions.
\end{abstract}

DOI: 10.1103/PhysRevB.88.115112

PACS number(s): 71.27.+a, 71.10.Fd

\section{INTRODUCTION}

Dynamical mean-field theory (DMFT) $)^{1,2}$ represents a big step forward for our understanding of strongly correlated electron systems. It fully includes local correlations, which often constitute the major contribution of electronic correlations. These are crucial for quasiparticle renormalization and the physics of the Mott-Hubbard metal-insulator transition (MIT). ${ }^{3}$ However, the arguably most fascinating phenomena of electronic correlations, such as unconventional superconductivity or (quantum) criticality, originate from (or at least are strongly affected by) nonlocal correlations. Hence, several approaches have been developed using DMFT as a starting point and including nonlocal correlations beyond. The two main routes to this end are cluster ${ }^{4-7}$ and diagrammatic extensions $^{8-12}$ of DMFT.

The basic idea of cluster extensions is to go beyond the single-site DMFT by extending the notion of locality to a cluster of sites. This way, correlations on length scales of the extension of the cluster, which hence are short ranged, are included. In practice, two different flavors are employed, which are essentially based on clusters in real or $\mathbf{k}$ space, and are coined cellular DMFT ${ }^{6}$ and dynamical cluster approximation (DCA), ${ }^{4}$ respectively. Complementarily, two distinct diagrammatic extensions of DMFT, based on the computation of the local two-particle vertex ${ }^{13}$ of the Anderson impurity model (AIM) associated to DMFT, have been proposed. Both diagrammatic schemes aim at the inclusion of short- and longrange nonlocal correlations, and share, to some extent, a similar philosophy ${ }^{14}$ with the diagrammatic treatments of the Anderson localization built around the infinite dimensional limit. ${ }^{15,16}$ The two diagrammatic extensions of DMFT, however, differ: the dynamical vertex approximation (DГA $)^{9,10,17}$ is based on the consideration of the fully two-particle irreducible local vertex, while the dual fermion (DF) ${ }^{11,12,18}$ diagrams are built from the two-particle local vertex which is one- and two-particle reducible.
The idea behind $\mathrm{DГА} \mathrm{is} \mathrm{a} \mathrm{systematic} \mathrm{resummation} \mathrm{of} \mathrm{the}$ most relevant Feynman diagrams beyond the DMFT ones: While DMFT is based on the locality of the fully irreducible one-particle vertex (i.e., the self-energy), DГА raises this locality concept to a higher level of the diagrammatics, requiring only the fully irreducible $n$-particle vertex to be local. Fortunately, there is compelling numerical evidence that, even in two dimensions, the fully irreducible $n=2$-particle vertex indeed is local, ${ }^{19}$ so that this vertex can be considered as a building block of the diagram technique in the DГA approach. The proper DГA treatment would hence correspond to the solution of the parquet equations, ${ }^{20}$ with an input given by the two-particle irreducible local vertex function. While the numerical solution of the parquet equations has been recently achieved ${ }^{21}$ for single-band two-dimensional models, the computational effort is still considerable. Hence, most of the DГА results obtained hitherto ${ }^{17,22}$ employed the ladder approximation, where, taking into account the most important channel(s) only, the assumption of locality is made for the two-particle vertices, which are irreducible in these channels.

The DF approach, instead, is a systematic functionalintegral expansion around DMFT. By introducing an impurity problem at each lattice site, the lattice action is recast in terms of decoupled impurities and a momentum-dependent remainder, which involves the hybridization function and the bare dispersion. Through a Hubbard-Stratonovich transformation acting on this term, so-called dual fermions are introduced. These couple only locally to the original fermionic degrees of freedom. Hence, the latter can be integrated out, which produces all local connected two- and moreparticle diagrams (vertices) of the impurity problem through which the dual fermions are coupled. This is in contrast with the ВГА which uses only the irreducible part of these vertices. ${ }^{23}$ Analogously to DГА, the inclusion and an exact treatment of all $n$-particle interaction terms among the electrons would correspond to the exact solution of the 
problem, but in practice three- and more-particle vertices are neglected and only the lowest-order interaction terms (i.e., the two-particle local vertex) for the dual electrons are considered. Different diagrammatic approximations within the DF approach, such as second-order perturbation theory, ${ }^{11}$ ladder series, ${ }^{12}$ and quite recently even parquet ${ }^{18}$ have been considered. We note here, that the DF parquet calculations, when performed without self-consistency, would be formally similar to the parquet corrections to the local physics of the Anderson localization problem considered in Ref. 15, though in the former case the local connected two-particle vertex is obviously much more complex than the one of CPA.

A thorough comparison between the diagrammatics of DF and $\mathrm{DГА} \mathrm{has} \mathrm{not} \mathrm{been} \mathrm{done} \mathrm{so} \mathrm{far,} \mathrm{although,} \mathrm{from} \mathrm{the} \mathrm{above}$ discussion one may surmise a sort of underlying similarity between the two diagrammatic approaches and their schemes of implementation. To perform such a comparison, we present in this paper a general approach for a systematic inclusion of nonlocal corrections beyond DMFT. This scheme is also based on a functional integral, similarly as in the DF approach, but it is formulated in terms of the one-particle irreducible (1PI) vertices instead of the reducible vertices of the DF approach. In order to illustrate the content of the 1PI approach we compare it diagrammatically and numerically with DF and DГ A, also illustrating the diagrammatic relations between the latter two approaches. Quite remarkably, our results demonstrate that the 1PI approach combines synergetically important features of the DF and DГA schemes.

In Sec. II we discuss the general structure of the nonlocal corrections to DMFT, considering contributions to the selfenergy, which are second order with respect to the bare on-site Coulomb repulsion and the DMFT local interaction, respectively. In Sec. III we develop a formalism based on the transformation to the one-particle irreducible functional in the DMFT part of the action. In Sec. IV we derive nonlocal contributions to the local (DMFT) self-energy based on ladder diagrams and discuss these in terms of a comparison with the DF and DГA approaches. In Sec. V, we discuss results for the two-dimensional Hubbard model obtained with our method, and, finally, Sec. VI is devoted to conclusions and an outlook.

\section{SECOND-ORDER PERTURBATION THEORY}

Let us briefly discuss the structure of the corrections to the dynamical mean-field theory by means of the perturbation theory. Specifically, we consider the Hubbard model with hopping $t_{i j}$ and Coulomb interaction $U$ :

$$
H=\sum_{i j \sigma} t_{i j} \hat{c}_{i \sigma}^{\dagger} \hat{c}_{j \sigma}+U \sum_{i} \hat{n}_{i \uparrow} \hat{n}_{i \downarrow} .
$$

Here, the operator $\hat{c}_{i \sigma}^{\dagger}\left(\hat{c}_{i \sigma}\right)$ creates (annihilates) an electron with spin $\sigma$ at lattice site $i, \hat{n}_{i \sigma}=\hat{c}_{i \sigma}^{\dagger} \hat{c}_{i \sigma}$. For the sake of simplicity, this paper deals with the one-band Hubbard model only, but a generalization of the 1PI approach to more complex multiorbital models is, as a matter of course, possible.
The dynamical mean-field theory approximates the corresponding full action by an effective local action ${ }^{2}$

$$
\begin{aligned}
\mathcal{S}_{\mathrm{DMFT}}\left[c^{+}, c\right]= & -\sum_{i} \frac{1}{\beta^{2}} \int_{0}^{\beta} d \tau \int_{0}^{\beta} d \tau^{\prime} \sum_{\sigma} c_{i \sigma}^{+}(\tau) \\
& \times \zeta^{-1}\left(\tau-\tau^{\prime}\right) c_{i \sigma}\left(\tau^{\prime}\right)+\int_{0}^{\beta} d \tau U n_{i \uparrow}(\tau) n_{i \downarrow}(\tau),
\end{aligned}
$$

where $c_{i \sigma}^{+}(\tau)$ and $c_{i \sigma}(\tau)$ are Grassmann variables corresponding to the Fermion operators $\hat{c}_{i \sigma}^{\dagger}$ and $\hat{c}_{i \sigma}$ at imaginary time $\tau, \beta=1 / T$ is the inverse temperature. The Weiss field, i.e., the noninteracting impurity Green's function $\zeta\left(\tau-\tau^{\prime}\right)$, has to be determined self-consistently in DMFT from the following condition on its Fourier transform $\zeta_{v}$

$$
\sum_{\mathbf{k}} G_{k}=\left(\zeta_{\nu}^{-1}-\Sigma_{\mathrm{loc}, v}\right)^{-1}=G_{\mathrm{loc}, v}^{-1}
$$

where

$$
G_{k}=\left(i v+\mu-\varepsilon_{\mathbf{k}}-\Sigma_{\mathrm{loc}, v}\right)^{-1}
$$

$\varepsilon_{\mathbf{k}}$ is the Fourier transform of $t_{i j}, \mu$ is the chemical potential, and $\Sigma_{\text {loc, } v}$ is the self-energy of the impurity problem [see Eq. (2)] at the fermionic Matsubara frequency $i v$ [i.e., $v=\frac{\pi}{\beta}(2 n+1), n \in \mathbb{Z}$ ]. Note that we specify all imaginary frequency arguments as subscripts (or, for the vertex functions below, as superscripts) and that we adopt a four-vector notation for the frequency and momentum arguments, i.e., $k=(v, \mathbf{k})$ for a fermionic and $q=(\omega, \mathbf{q})$ for a bosonic Matsubara frequency [i.e., $\omega=\frac{\pi}{\beta}(2 m), m \in \mathbb{Z}$ ]. The subscript "loc" is attached to all quantities (Green's functions and vertices) of the local AIM despite the Weiss fields $\zeta_{v}$. In practice, the local problem in Eq. (2) is solved numerically by exact diagonalization or quantum Monte Carlo simulations, ${ }^{2}$ yielding a self-energy $\Sigma_{\text {loc, } v}$ and Green's function $G_{\text {loc, } v}$ until self-consistency regarding Eq. (3) is obtained. Since such numerical calculations can be better performed in Matsubara frequencies, we stick to this formalism, but a transformation to real frequencies is possible.

DMFT takes into account local dynamical correlations but it neglects intersite correlations, which is reflected in a wave vector k-independent self-energy $\Sigma_{k} \equiv \Sigma_{\text {loc, }, ~}$. Perturbation theories such as self-consistent $T$ matrix, fluctuation exchange (FLEX), and parquet approximation ${ }^{24}$ can result in a $\mathbf{k}$ dependent $\Sigma$, but the most important local correlations are not reliably reproduced when the system is not in the weak coupling regime, i.e., if the Coulomb interaction parameter $U$ is comparable to or larger than the bandwidth.

To illustrate the structure of nonlocal corrections beyond DMFT, we first analyze the perturbation theory. Since we want to find corrections to the already calculated local (DMFT) selfenergy we use the DMFT Green's function, given in Eq. (4), as bare propagator for the construction of self-energy diagrams. Let us now separate purely local and nonlocal contributions to $\Sigma_{k}$ by introducing the function

$$
\widetilde{G}_{k} \equiv G_{k}-G_{\mathrm{loc}, v},
$$


which vanishes after averaging in $\mathbf{k}$ space by construction:

$$
\sum_{\mathbf{k}} \widetilde{G}_{k}=0
$$

In the second order in $U$ we obtain for the nonlocal self-energy:

$$
\begin{aligned}
\Sigma_{k}^{(2)} & =\frac{U^{2}}{\beta} \sum_{q} G_{k-q} V_{q}^{(2)}=\Sigma_{\mathrm{loc}, \nu}^{(2)}+\widetilde{\Sigma}_{k}^{(2)} \\
\Sigma_{\mathrm{loc}, \nu}^{(2)} & =\frac{U^{2}}{\beta} \sum_{\omega} G_{\mathrm{loc}, \nu-\omega} V_{\mathrm{loc}, \omega}^{(2)} \\
\widetilde{\Sigma}_{k}^{(2)} & =\frac{U^{2}}{\beta} \sum_{q} \widetilde{G}_{k-q} \widetilde{V}_{q}^{(2)},
\end{aligned}
$$

where

$$
\begin{aligned}
V_{q}^{(2)} & =-\frac{1}{\beta} \sum_{k^{\prime}} G_{k^{\prime}+q} G_{k^{\prime}}=V_{\mathrm{loc}, \omega}^{(2)}+\widetilde{V}_{q}^{(2)}, \\
V_{\mathrm{loc}, \omega}^{(2)} & =\sum_{\nu^{\prime}} \chi_{\mathrm{loc}}^{0, \nu^{\prime} \omega}, \quad \widetilde{V}_{q}^{(2)}=\sum_{\nu^{\prime}} \widetilde{\chi}_{q}^{v^{\prime}},
\end{aligned}
$$

and $\chi_{\text {loc }}^{0, v \omega}$ and $\tilde{\chi}_{q}^{v}$ are defined as

$$
\begin{aligned}
\chi_{\mathrm{loc}}^{0, v \omega} & =-\frac{1}{\beta} G_{\mathrm{loc}, \nu} G_{\mathrm{loc}, \nu+\omega}, \\
\tilde{\chi}_{q}^{v} & =-\frac{1}{\beta} \sum_{\mathbf{k}} \widetilde{G}_{k} \widetilde{G}_{k+q} .
\end{aligned}
$$

The mixed local-nonlocal terms in Eq. (7) vanish due to the identity in Eq. (6). For the same reason $\widetilde{V}_{q}^{(2)}$ vanishes after averaging in $\mathbf{k}$ space:

$$
\sum_{\mathbf{q}} \widetilde{V}_{q}^{(2)}=-\frac{1}{\beta} \sum_{k^{\prime}}\left\{\sum_{\mathbf{q}} \widetilde{G}_{k^{\prime}+q}\right\} \widetilde{G}_{k^{\prime}}=0 .
$$

The local part $\Sigma_{\text {loc,v }}$ in Eq. (7) can be replaced by its DMFT value, so that only nonlocal terms are calculated by perturbation theory.

In higher orders of the perturbation theory, there is no such clear separation of terms: mixed local-nonlocal terms appear in $\Sigma_{k}$. Considering, however, the leading nonlocal correction to the local self-energy, these terms can be reduced to those containing the local vertex instead of $U$ in the secondorder result, Eq. (7), and the nonlocal part of the Green's functions. In particular, using the dual fermion approach ${ }^{11}$ the corresponding correction can be expressed as

$$
\Sigma_{\mathrm{d}, k}^{(2)}=\frac{1}{2 \beta} \sum_{\nu^{\prime}, q} \sum_{m=c, s} A_{m} \Gamma_{\mathrm{loc}, m}^{\nu v^{\prime} \omega} \tilde{\chi}_{q}^{v^{\prime}} \Gamma_{\mathrm{loc}, m}^{\nu^{\prime} v \omega} G_{k+q},
$$

where $A_{s}=3 / 2 ; A_{c}=1 / 2, \quad \Gamma_{\operatorname{loc}, s(c)}^{\nu v^{\prime} \omega}=-\Gamma_{\operatorname{loc}, \uparrow \uparrow}^{\nu \nu^{\prime} \omega} \pm \Gamma_{\operatorname{loc}, \uparrow \downarrow}^{\nu v^{\prime} \omega}$ is the local two-particle vertex, which is related to the local susceptibility

$$
\begin{aligned}
\chi_{\text {loc }, \sigma \sigma^{\prime}}^{\nu v^{\prime} \omega}= & \frac{1}{\beta^{2}} \int_{0}^{\beta} d \tau_{1} d \tau_{2} d \tau_{3} \mathrm{e}^{-i \tau_{1} v} \mathrm{e}^{i \tau_{2}(v+\omega)} \mathrm{e}^{-i \tau_{3}\left(\nu^{\prime}+\omega\right)} \\
& \times\left[\left\langle T_{\tau} \hat{c}_{i \sigma}^{\dagger}\left(\tau_{1}\right) \hat{c}_{i \sigma}\left(\tau_{2}\right) \hat{c}_{i \sigma^{\prime}}^{\dagger}\left(\tau_{3}\right) \hat{c}_{i \sigma^{\prime}}(0)\right\rangle\right. \\
& \left.-\left\langle T_{\tau} \hat{c}_{i \sigma}^{\dagger}\left(\tau_{1}\right) \hat{c}_{i \sigma}\left(\tau_{2}\right)\right\rangle\left\langle T_{\tau} \hat{c}_{i \sigma^{\prime}}^{\dagger}\left(\tau_{3}\right) \hat{c}_{i \sigma^{\prime}}(0)\right\rangle\right]
\end{aligned}
$$

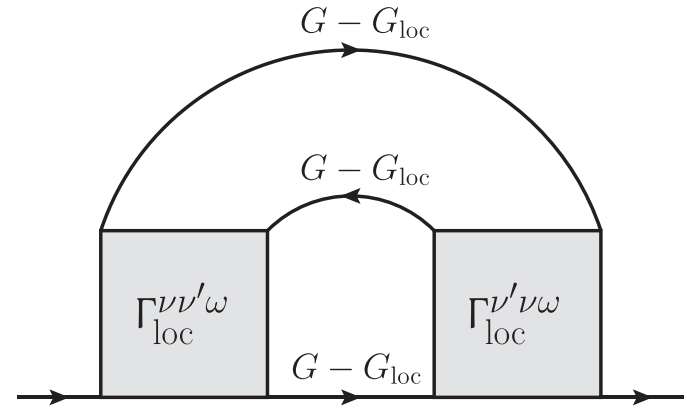

FIG. 1. Second-order diagram for the DF approach in terms of real electrons.

by

$$
\Gamma_{\mathrm{loc}, \sigma \sigma^{\prime}}^{\nu \nu^{\prime} \omega}=-\frac{\chi_{\mathrm{loc}, \sigma \sigma^{\prime}}^{v v^{\prime} \omega}-\chi_{\mathrm{loc}}^{0, v \omega} \delta_{\nu v^{\prime}} \delta_{\sigma \sigma^{\prime}}}{\chi_{\mathrm{loc}}^{0, v \omega} \chi_{\mathrm{loc}}^{0, v^{\prime} \omega}}
$$

The susceptibilities $\chi_{\mathrm{loc}, \sigma \sigma^{\prime}}^{\nu \nu^{\prime} \omega}$ can be obtained from the exact diagonalization or quantum Monte Carlo solution of the single-impurity problem. The result (11) is illustrated diagrammatically in Fig. 1.

In the DF approach the self-energy $\Sigma_{\mathrm{d}, \mathrm{k}}$ is however an auxiliary construct. It is related to the real self-energy $\Sigma_{k}$ of the system via

$$
\Sigma_{k}=\Sigma_{\mathrm{DF}, k}=\frac{\Sigma_{\mathrm{d}, k}}{1+G_{\mathrm{loc}, v} \Sigma_{\mathrm{d}, k}}+\Sigma_{\mathrm{loc}, v} .
$$

In order to understand this equation diagrammatically, one can expand the denominator in a geometric series. This procedure generates, together with $\Sigma_{\mathrm{d}}$ from Fig. 1, the additional diagram depicted in Fig. 2 and chain expansions thereof. Evidently, the lattice self-energy should not contain one-particle reducible Feynman diagrams. Indeed, as discussed in Ref. 23, including three- and more-particle vertices in the DF calculations actually removes these spurious contributions to the self-energy. In our example, the diagram shown in Fig. 2 is canceled by a corresponding contribution from the one-particle reducible three-particle vertex (shown in red in Fig. 2).

The above-discussed difficulty is obviously not a property of the DF approach per se but its truncation at the two-particle vertex level while keeping, at the same time, the full denominator of Eq. (14). This is analogous to the linked cluster theorem, as a consequence of which all vacuum-to-vacuum diagrams cancel in the ratio of path integrals, which appears in the calculation of correlation functions. Of course, this is no longer true if we perform an expansion of the path integral in the numerator only up to a certain order. Similarly, if we truncate the DF approach at the two-particle vertex level, reducible diagrams stemming from local one-particle reducible threeand more-particle vertex functions are not present and, hence, the denominator in Eq. (14) introduces such terms in the diagrammatic expansion rather than canceling them. To avoid these complications as well as to account systematically for the contribution of one-particle reducible diagrams to threeand more-particle vertices, we consider below the one-particle irreducible formulation of the generating functional approach. 


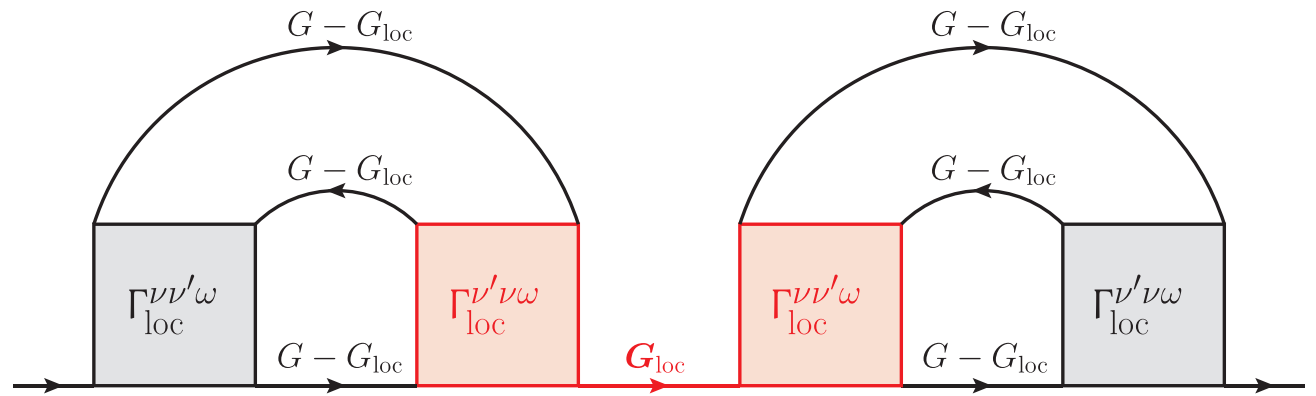

FIG. 2. (Color online) A reducible diagram for the real self-energy $\Sigma_{k}$ stemming from the expansion of the denominator in Eq. (14) and the diagram of Fig. 1 as numerator. In the DF approach, the same contribution, albeit with opposite sign, is generated from a diagram involving the three-particle vertex, which contains the part marked in red, hence canceling this reducible contribution.

\section{ONE-PARTICLE IRREDUCIBLE (1PI) APPROACH}

For a general formulation of the nonlocal corrections to DMFT we separate the nonlocal degrees of freedom in the generating functional formalism. To this end, we consider the generating functional

$$
Z\left[\eta^{+}, \eta\right]=\int D\left[c^{+}, c\right] \exp \left\{-\int_{0}^{\beta} d \tau\left[\sum_{i, \sigma}\left(c_{i \sigma}^{+}(\tau) \frac{\partial c_{i \sigma}(\tau)}{\partial \tau}-\eta_{i \sigma}^{+}(\tau) c_{i \sigma}(\tau)-c_{i \sigma}^{+}(\tau) \eta_{i \sigma}(\tau)\right)+H\left[c^{+}, c\right]\right]\right\},
$$

where $\eta_{i \sigma}^{+}(\tau), \eta_{i \sigma}(\tau)$ are the fermionic source fields. The contribution of the local and nonlocal degrees of freedom can be split by performing a Fourier transform in the exponent and introducing the auxiliary fields $\widetilde{c}^{+}$and $\widetilde{c}:{ }^{25}$

$$
\begin{aligned}
Z\left[\eta^{+}, \eta\right]= & \int D\left[\widetilde{c}^{+}, \widetilde{c}\right] \exp \left\{\beta \sum_{k, \sigma} \widetilde{c}_{k \sigma}^{+}\left(\zeta_{\nu}^{-1}-G_{0 k}^{-1}\right)^{-1} \widetilde{c}_{k \sigma}\right\} \\
& \times \int D\left[c^{+}, c\right] \exp \left\{-\mathcal{S}_{\mathrm{DMFT}}\left[c^{+}, c\right]+\sum_{k, \sigma}\left[\left(\eta_{k \sigma}^{+}+\widetilde{c}_{k \sigma}^{+}\right) c_{k \sigma}+c_{k \sigma}^{+}\left(\eta_{k \sigma}+\widetilde{c}_{k \sigma}\right)\right]\right\},
\end{aligned}
$$

where $G_{0 k}^{-1}=i \nu-\varepsilon_{\mathbf{k}}$ is the noninteracting lattice Green's function. Let us recall that the correlation (or Green's) functions can be obtained by functional derivatives of $\log Z\left[\eta^{+}, \eta\right]$ with respect to $\eta^{+}$and $\eta$ at $\eta^{+}=\eta=0$, which allows us to neglect any normalization factor which would appear in front of the integral on the right-hand side of Eq. (16).

Whereas up to this point the formalism is essentially the same as in the derivation of the DF approach, ${ }^{11,26}$ we now apply a Legendre transform in order to pass to the 1PI functional in the DMFT part of the action

$$
\begin{aligned}
\exp \left(-W_{\mathrm{DMFT}}\left[\widetilde{\eta}^{+}, \widetilde{\eta}\right]\right) & =\int D\left[c^{+}, c\right] \exp \left\{-\mathcal{S}_{\mathrm{DMFT}}\left[c^{+}, c\right]+\sum_{k, \sigma}\left(\widetilde{\eta}_{k \sigma}^{+} c_{k \sigma}+c_{k \sigma}^{+} \widetilde{\eta}_{k \sigma}\right)\right\} \\
& =\exp \left\{-\Gamma_{\mathrm{DMFT}}\left[\phi^{+}, \phi\right]+\sum_{k, \sigma}\left(\widetilde{\eta}_{k \sigma}^{+} \phi_{k \sigma}+\phi_{k \sigma}^{+} \widetilde{\eta}_{k \sigma}\right)\right\},
\end{aligned}
$$

where

$$
\phi_{k \sigma}=-\frac{\delta W_{\mathrm{DMFT}}\left[\widetilde{\eta}^{+}, \widetilde{\eta}\right]}{\delta \widetilde{\eta}_{k \sigma}^{+}}, \quad \widetilde{\eta}_{k \sigma}=\frac{\delta \Gamma_{\mathrm{DMFT}}\left[\phi^{+}, \phi\right]}{\delta \phi_{k \sigma}^{+}},
$$

$\widetilde{\eta}_{k \sigma}=\eta_{k \sigma}+\widetilde{c}_{k \sigma}$, and similarly [but with reversed sign in Eq. (18)] for the conjugated fields. Therefore, Eq. (16) becomes

$$
Z\left[\eta^{+}, \eta\right]=\int D\left[\widetilde{c}^{+}, \widetilde{c}\right] \exp \left\{\beta \sum_{k, \sigma} \widetilde{c}_{k \sigma}^{+}\left(\zeta_{\nu}^{-1}-G_{0 k}^{-1}\right)^{-1} \widetilde{c}_{k \sigma}+\sum_{k, \sigma}\left[\left(\eta_{k \sigma}^{+}+\widetilde{c}_{k \sigma}^{+}\right) \phi_{k \sigma}+\phi_{k, \sigma}^{+}\left(\eta_{k \sigma}+\widetilde{c}_{k \sigma}\right)\right]-\Gamma_{\mathrm{DMFT}}\left[\phi^{+}, \phi\right]\right\} .
$$


The fields $\phi^{+}$and $\phi$ in this representation are functionals of the fields $\widetilde{\eta}^{+}$and $\widetilde{\eta}$ defined via the relations in Eq. (18). For the following consideration it is convenient to change the variables of integration from $\widetilde{c}^{+}, \widetilde{c}$ to $\phi^{+}, \phi$. This yields

$$
\begin{aligned}
Z\left[\eta^{+}, \eta\right]= & \int D\left[\phi^{+}, \phi\right] \exp \left\{\beta \sum_{k, \sigma}\left(\frac{\delta \Gamma_{\mathrm{DMFT}}\left[\phi^{+}, \phi\right]}{\delta \phi_{k \sigma}}+\eta_{k \sigma}^{+}\right)\left[\zeta_{v}^{-1}-G_{0 k}^{-1}\right]^{-1}\left(-\frac{\delta \Gamma_{\mathrm{DMFT}}\left[\phi^{+}, \phi\right]}{\delta \phi_{k \sigma}^{+}}+\eta_{k \sigma}\right)\right. \\
& \left.-\sum_{k, \sigma}\left(\frac{\delta \Gamma_{\mathrm{DMFT}}\left[\phi^{+}, \phi\right]}{\delta \phi_{k \sigma}} \phi_{k \sigma}-\phi_{k \sigma}^{+} \frac{\delta \Gamma_{\mathrm{DMFT}}\left[\phi^{+}, \phi\right]}{\delta \phi_{k \sigma}^{+}}\right)-\Gamma_{\mathrm{DMFT}}\left[\phi^{+}, \phi\right]\right\} J\left[\phi^{+}, \phi\right],
\end{aligned}
$$

where $J^{-1}\left[\phi^{+}, \phi\right]=\operatorname{det} \delta^{2} \Gamma_{\mathrm{DMFT}} /\left(\delta \phi^{+} \delta \phi\right)$ is the determinant of the Jacobian of the corresponding transformation, see Appendix for more details.

We proceed now by expanding the functional $\Gamma_{\mathrm{DMFT}}\left[\phi^{+}, \phi\right]$ into a series with respect to the source fields $\phi^{+}$and $\phi$. In the DF approach such an expansion is performed for the functional $W_{\mathrm{DMFT}}\left[\eta^{+}, \eta\right]$, which generates connected but in general one-particle reducible vertex functions as the coefficients of this expansion. Expanding $\Gamma_{\text {DMFT }}$ instead, one obtains the (local) one-particle irreducible vertex functions amputated by the outer legs. Neglecting the constant zeroth-order contribution, the resulting expansion up to fourth order, i.e., up to the level of the two-particle vertex function, reads

$$
\Gamma_{\mathrm{DMFT}}\left[\phi^{+}, \phi\right]=-\frac{1}{\beta} \sum_{k, \sigma} G_{\mathrm{loc}, \nu}^{-1} \phi_{k \sigma}^{+} \phi_{k \sigma}+\frac{1}{2 \beta^{3}} \sum_{k k^{\prime} q} \sum_{\sigma \sigma^{\prime}} \widetilde{\Gamma}_{\mathrm{loc}, \sigma \sigma^{\prime}}^{\nu \nu^{\prime} \omega}\left(\phi_{k \sigma}^{+} \phi_{k+q, \sigma}\right)\left(\phi_{k^{\prime}+q, \sigma^{\prime}}^{+} \phi_{k^{\prime} \sigma^{\prime}}\right),
$$

where $\widetilde{\Gamma}_{\text {loc, } \sigma \sigma^{\prime}}^{v \nu^{\prime}}=\left(1-\frac{1}{2} \delta_{\sigma \sigma^{\prime}}\right) \Gamma_{\text {loc, } \sigma \sigma^{\prime}}^{v v^{\prime} \omega}$

In the next step, we use the (approximate) DMFT functional $\Gamma_{\text {DMFT }}$ from Eq. (21) for evaluating Eq. (20), i.e., we have to calculate the derivatives of the functional $\Gamma_{\text {DMFT }}$ with respect to the fields $\phi^{+}$and $\phi$. While the formal derivation is given in Appendix, let us here discuss the most important features of the calculation. The exponent in Eq. (20) contains a term proportional to $\left(\delta_{\phi} \Gamma_{\text {DMFT }}\right)\left(\delta_{\phi^{+}} \Gamma_{\text {DMFT }}\right.$ ) (where $\delta_{\phi}$ denotes the functional derivative with respect to the field $\phi$ ). Inserting now the two-particle part of $\Gamma_{\text {DMFT }}$ into this expression clearly leads to a term which is proportional to $\left(\Gamma_{\text {loc }}\right)^{2}\left(\phi^{+} \phi\right)^{3}$. Such a contribution stems from the local reducible three- (and more)-particle vertices, and is hence absent in the DF approach if we neglect these vertices. At the same time, such contributions stemming from reducible (local) diagrams are fully taken into account in the 1PI approach when expanding $\Gamma_{\text {DMFT }}$ up to the two-particle level. The above mentioned three-particle contribution can be decoupled by another Hubbard-Stratonovich transformation introducing new fields $\psi^{+}$and $\psi$. The corresponding calculations are carried out in Appendix and yield

$$
\begin{aligned}
Z\left[\eta^{+}, \eta\right]= & \int D\left[\phi^{+}, \phi\right] D\left[\psi^{+}, \psi\right] \exp \left\{\sum_{k, \sigma} \eta_{k \sigma}^{+}\left(\psi_{k \sigma}+\phi_{k \sigma}\right)+\left(\psi_{k \sigma}^{+}+\phi_{k \sigma}^{+}\right) \eta_{k \sigma}\right. \\
& +\frac{1}{\beta} \sum_{k, \sigma} G_{k}^{-1}\left(\phi_{k \sigma}^{+} \phi_{k \sigma}+\psi_{k \sigma}^{+} \phi_{k \sigma}+\phi_{k \sigma}^{+} \psi_{k \sigma}\right)+\left(G_{k}^{-1}-G_{\mathrm{loc}, v}^{-1}\right) \psi_{k \sigma}^{+} \psi_{k \sigma} \\
& -\frac{1}{\beta^{3}} \sum_{k k^{\prime} q} \sum_{\sigma \sigma^{\prime}} \widetilde{\Gamma}_{\mathrm{loc}, \sigma \sigma^{\prime}}^{\nu v^{\prime} \omega}\left[\left(\psi_{k \sigma}^{+} \phi_{k+q, \sigma}\right)\left(\phi_{k^{\prime}+q, \sigma^{\prime}}^{+} \phi_{k^{\prime} \sigma^{\prime}}\right)\right. \\
& \left.\left.+\left(\phi_{k \sigma}^{+} \phi_{k+q, \sigma}\right)\left(\phi_{k^{\prime}+q, \sigma^{\prime}}^{+} \psi_{k^{\prime} \sigma^{\prime}}\right)+\frac{1}{2}\left(\phi_{k \sigma}^{+} \phi_{k+q, \sigma}\right)\left(\phi_{k^{\prime}+q, \sigma^{\prime}}^{+} \phi_{k^{\prime} \sigma^{\prime}}\right)\right]\right\} J\left[\phi^{+}, \phi\right],
\end{aligned}
$$

where $G_{k}$ is defined by Eq. (4) and accounts for the local self-energy. Equation (22) expresses the partition function through the one-particle irreducible local vertex $\Gamma_{\operatorname{loc}, \sigma \sigma^{\prime}}^{v \nu^{\prime} \omega}$ and the local self-energy, and represents one of the central results of the present paper. The first line of Eq. (22) includes the source fields, the second line contains the quadratic (bare) terms in fermionic fields, and the last two lines correspond to the interaction between fermionic degrees of freedom. The nonlocal Green's functions of the lattice model is defined as $\mathbb{G}_{k \sigma}=-\frac{1}{\beta}\left\langle\left\langle c_{k \sigma} \mid c_{k \sigma}^{+}\right\rangle\right\rangle$. It can be calculated through derivatives of Eq. (22) with respect to the source fields $\eta^{+}, \eta$ :

$$
\begin{aligned}
\mathbb{G}_{k \sigma}= & \left.\frac{1}{\beta} \frac{\delta^{2} \ln Z}{\delta \eta_{k \sigma}^{+} \delta \eta_{k \sigma}}\right|_{\eta^{+}=\eta=0} \\
= & -\frac{1}{\beta}\left[\left\langle\left\langle\phi_{k \sigma} \mid \phi_{k \sigma}^{+}\right\rangle\right\rangle+\left\langle\left\langle\phi_{k \sigma} \mid \psi_{k \sigma}^{+}\right\rangle\right\rangle+\left\langle\left\langle\psi_{k \sigma} \mid \phi_{k \sigma}^{+}\right\rangle\right\rangle\right. \\
& \left.+\left\langle\left\langle\psi_{k \sigma} \mid \psi_{k \sigma}^{+}\right\rangle\right\rangle\right] .
\end{aligned}
$$

That is, $\mathbb{G}_{k \sigma}$ can be written as the sum of four distinct propagators, which can be combined in a more compact form $-\frac{1}{\beta} \sum_{a, b=1,2}\left\langle\left\langle\Phi_{k \sigma}^{a} \mid \Phi_{k \sigma}^{+, b}\right\rangle\right\rangle$, where we have introduced a spinor

$$
\Phi_{k \sigma}=\left(\begin{array}{c}
\phi_{k \sigma} \\
\psi_{k \sigma}
\end{array}\right)
$$

In order to treat the interaction in Eq. (22), we consider first the bare part in the action, which is quadratic in Grassmann variables. The corresponding 1PI bare propagators are obtained by setting $\Gamma_{\text {loc }}=0$ in Eq. (22) and yield

$$
\begin{aligned}
\mathcal{G}_{k} & =-\frac{1}{\beta}\left\langle\left\langle\Phi_{k} \mid \Phi_{k}^{+}\right\rangle\right\rangle_{0}=\left(\begin{array}{cc}
G_{k}^{-1} & G_{k}^{-1} \\
G_{k}^{-1} & G_{k}^{-1}-G_{\mathrm{loc}, v}^{-1}
\end{array}\right)^{-1} \\
& =\left(\begin{array}{cc}
G_{k}-G_{\mathrm{loc}, v} & G_{\mathrm{loc}, v} \\
G_{\mathrm{loc}, v} & -G_{\mathrm{loc}, v}
\end{array}\right) .
\end{aligned}
$$


(a)

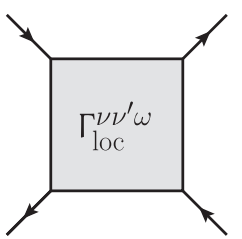

$\left(\phi^{+} \phi\right)\left(\phi^{+} \phi\right)$

(b)

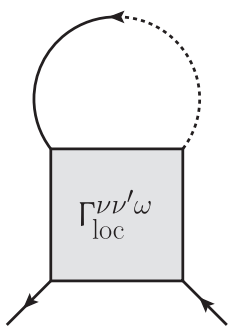

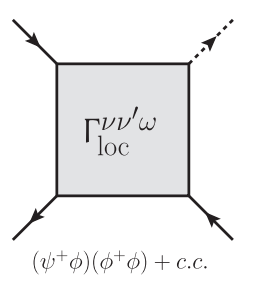

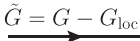

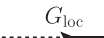

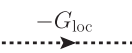

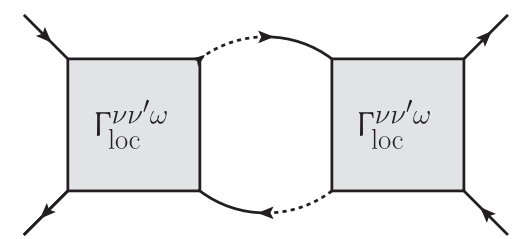

FIG. 3. (a) Elements of the diagram technique in the 1PI approach. (b) Diagrams that are generated by the elements in (a) but excluded (canceled) by the corresponding counterterms in the (inverse) determinant $J\left[\phi^{+}, \phi\right]$ of the Jacobian (see Appendix).

Let us again stress that the bare functions $G_{k}$ and $G_{\text {loc, } v}$ include all local self-interaction effects via the local self-energy $\Sigma_{\text {loc, } \nu}$, which is already considered in the DMFT part of the action [see Eqs. (16) and (17)]. The propagator $-\frac{1}{\beta}\left\langle\left\langle\phi_{k} \mid \phi_{k}^{+}\right\rangle\right\rangle_{0} \equiv \widetilde{G}_{k}=G_{k}-G_{\text {loc, }, ~}$ [as defined in Eq. (5)] corresponds to the remaining nonlocal fluctuations and obeys $\sum_{\mathbf{k}} \widetilde{G}_{k}=0$. The propagators $-\frac{1}{\beta}\left\langle\left\langle\phi_{k} \mid \psi_{k}^{+}\right\rangle\right\rangle_{0}=$ $-\frac{1}{\beta}\left\langle\left\langle\psi_{k} \mid \phi_{k}^{+}\right\rangle\right\rangle_{0}=\frac{1}{\beta}\left\langle\left\langle\psi_{k} \mid \psi_{k}^{+}\right\rangle\right\rangle_{0}=G_{\text {loc, } v}$ describe the (bare) local quasiparticles, coupled to the nonlocal degrees of freedom via the interaction in the third line in Eq. (22). The corresponding elements of the diagram technique are shown in Fig. 3(a). The interaction term consists of two parts, which are depicted diagrammatically. The first diagram corresponds to the contribution $\Gamma_{\text {loc }}\left(\phi^{+} \phi\right)\left(\phi^{+} \phi\right)$ in the fourth line of Eq. (22). This vertex can be either coupled to both local $\left(\left\langle\left\langle\phi \mid \psi^{+}\right\rangle\right\rangle\right.$or $\left.\left\langle\left\langle\psi \mid \phi^{+}\right\rangle\right\rangle\right)$and nonlocal propagators $\left(\left\langle\left\langle\phi \mid \phi^{+}\right\rangle\right\rangle\right)$or to nonlocal propagators only. In contrast, the other mentioned contribution to the interaction $\Gamma_{\text {loc }}\left(\psi^{+} \phi\right)\left(\phi^{+} \phi\right)+$ c.c. [third row of Eq. (22) and second diagram in Fig. 3(a)] is connected to at least one local propagator. Finally, the determinant $J\left[\phi^{+}, \phi\right]$ provides for the subtraction of diagrams which are already accounted for in $\Sigma_{\text {loc }}$ and $\Gamma_{\text {loc }}$, in particular the bubbles with one (i.e., tadpole terms) and two local Green's functions, which should be excluded from the diagram technique, see Fig. 3(b) and Appendix for details.

Let us finally comment on the the analytic properties of our approach: From the diagrammatic elements of the 1PI method in Fig. 3 one can infer that the situation is completely equivalent to the DF case. For the DF approach, the analyticity of the self-energy has been proven in Ref. 27. For a complete proof, it is however necessary to show that the corresponding statement holds for the Green's function as well, which remains an open problem. We note that, in practice, no causality violations have been observed in DF and hence we also do not expect violations in our practical calculations.

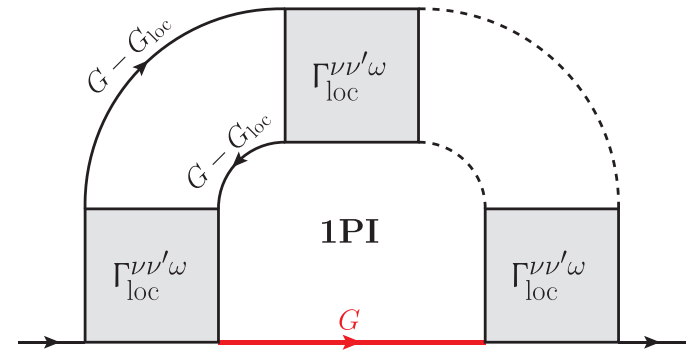

FIG. 4. (Color online) Third-order (in terms of the local vertex $\left.\Gamma_{\text {loc, } \sigma \sigma^{\prime}}^{v v^{\prime}}\right)$ self-energy diagram in the 1PI scheme, and ladder extension thereof (indicated by the dashed lines).

\section{LADDER APPROXIMATION IN THE 1PI APPROACH}

Aiming at a practical application of the 1PI scheme derived in Sec. III, we will now explicitly consider ladder diagrams for Eq. (22), see Fig. 4. As we mentioned in the Introduction, the restriction to ladder diagrams is, de facto, the typical approximation scheme adopted in the other diagrammatic extensions of DMFT. Hence, it represents the natural framework for testing the validity of the 1PI scheme and for comparing its diagrammatic and physical content against that of DF and DГA.

As for the explicit derivation of the corresponding 1PI expressions for the ladder diagrams, we start from the analysis of all possible bubble diagrams, which can be constructed from the diagrammatic elements for the 1PI approach discussed in the previous section (see Fig. 3). Considering all possible bubbles (Fig. 5), we observe that the bubble [Fig. 5(a)] with two local Green's functions should not appear in our 1PI corrections to the local self-energy, since it is already included in $\Gamma_{\text {loc }}$ [the contribution of Fig. 5(a) is canceled by the corresponding counterterms contained in the determinant $J\left[\phi^{+}, \phi\right]$ of the Jacobian, shown by the second diagram of Fig. 3(b)]. On the other hand, bubble diagrams with a single local Green's function, as depicted in Fig. 5(b) and 5(c) vanish due to the fact that $\widetilde{G}_{k}$, summed over $\mathbf{k}$, yields zero. Hence, the ladder part of the diagram for the self-energy can be solely composed of bubbles with two nonlocal Green's functions $\widetilde{G}_{k}$ [see Fig. 5(d)], which makes the considered approach similar to that in Ref. 14 with the restriction to the ladder diagrams only. Therefore, the ladder part has to be constructed solely from $\Gamma_{\text {loc }}\left(\phi^{+} \phi\right)\left(\phi^{+} \phi\right)$ vertices, except for the leftmost and rightmost vertex, which can be either of the (a)

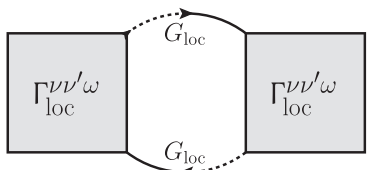

(c)

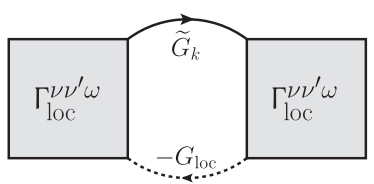

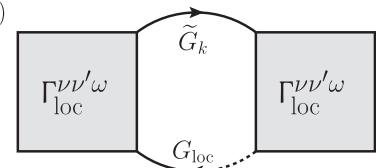

(d)

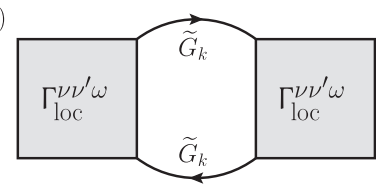

FIG. 5. Bubble diagrams for the 1PI approach which can be constructed from the diagrammatic elements shown in Fig. 3. Only the diagram (d) contributes to the perturbation series. 

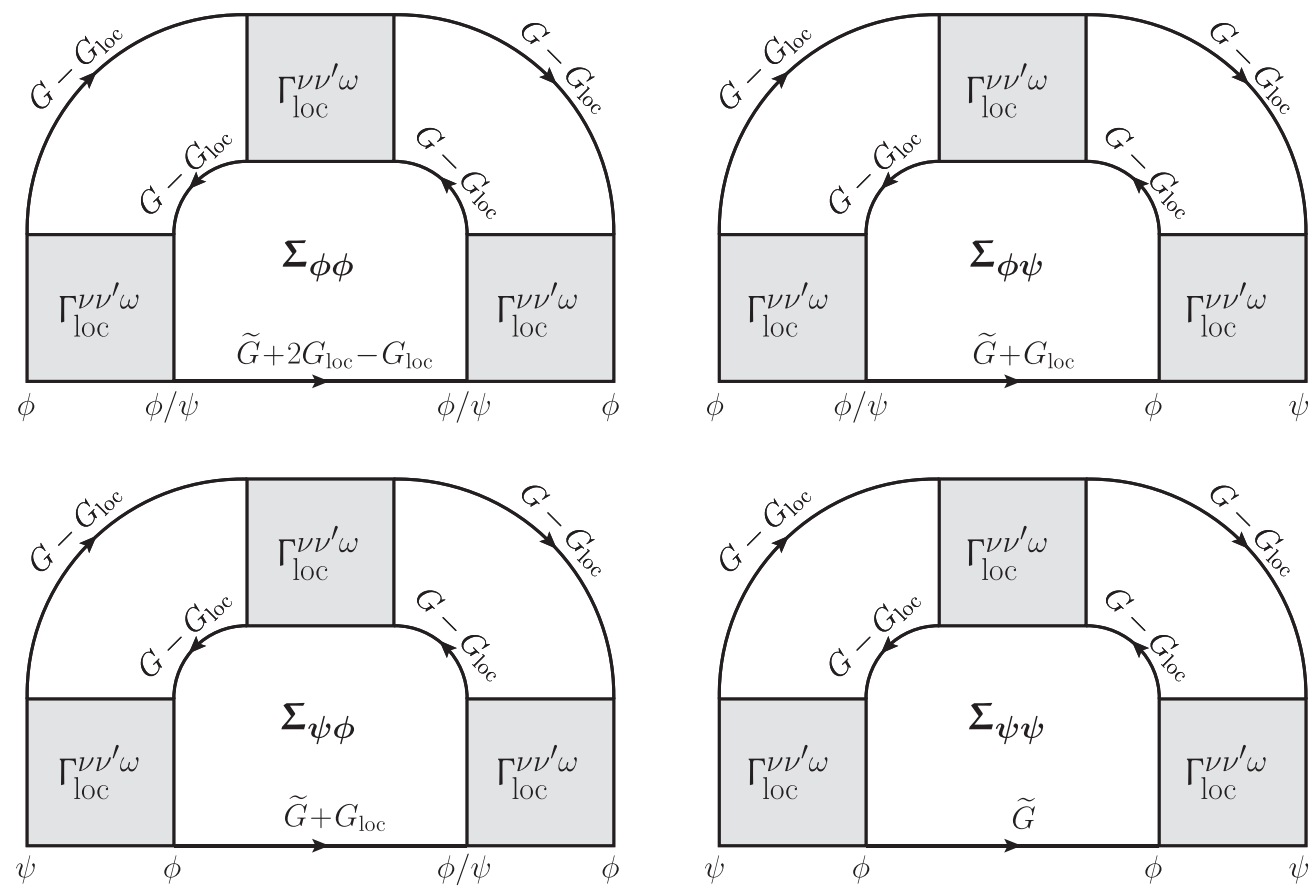

FIG. 6. The four components of the matrix $\Sigma$ depicted for diagrams of third order in $\Gamma_{\text {loc }}$.

type $\Gamma_{\text {loc }}\left(\psi^{+} \phi+\phi^{+} \psi\right)\left(\phi^{+} \phi\right)$, connected by one local Green's function $G_{\text {loc }}$, or of the type $\Gamma_{\text {loc }}\left(\phi^{+} \phi\right)\left(\phi^{+} \phi\right)$, connected by the Green's function $\widetilde{G}_{k}$. Hence, as it is illustrated in Fig. 6 for the case of third-order (in $\Gamma_{\text {loc }}$ ) diagrams, the self-energy matrix in the spinor representation,

$$
\boldsymbol{\Sigma}=\left(\begin{array}{cc}
\Sigma_{\phi \phi} & \Sigma_{\phi \psi} \\
\Sigma_{\psi \phi} & \Sigma_{\psi \psi}
\end{array}\right),
$$

has only two distinct components:

$$
\begin{aligned}
\Sigma_{\phi \phi, k} & =\Sigma_{\phi \psi, k}=\Sigma_{\psi \phi, k}=\Sigma_{1, k}+\Sigma_{2, k}, \\
\Sigma_{\psi \psi, k} & =\Sigma_{1, k},
\end{aligned}
$$

with $\Sigma_{1, k}$ and $\Sigma_{2, k}$ being defined in the following way:

$$
\begin{aligned}
\Sigma_{1, k}= & \frac{1}{\beta} \sum_{v^{\prime} v^{\prime \prime} q} \sum_{m=c, s} A_{m} \Gamma_{\mathrm{loc}, m}^{\nu v^{\prime} \omega}\left[\delta_{v^{\prime} v^{\prime \prime}}-\tilde{\chi}_{q}^{v^{\prime}} \Gamma_{\mathrm{loc}, m}^{\nu^{\prime} v^{\prime \prime} \omega}\right]_{v^{\prime} v^{\prime \prime}}^{-1} \\
& \times \widetilde{\chi}_{q}^{\nu^{\prime \prime}}\left[G_{k+q}-G_{\mathrm{loc}, v+\omega}\right] \Gamma_{\mathrm{loc}, m}^{\nu^{\prime \prime} \nu \omega}-\Sigma_{1, k}^{(2)}
\end{aligned}
$$

contains the $\widetilde{G}_{k}$ Green's functions only and

$$
\begin{aligned}
\Sigma_{2, k}= & \frac{1}{\beta} \sum_{v^{\prime} v^{\prime \prime} q} \sum_{m=c, s} A_{m} \Gamma_{\mathrm{loc}, m}^{\nu v^{\prime} \omega}\left[\delta_{v^{\prime} v^{\prime \prime}}-\tilde{\chi}_{q}^{v^{\prime}} \Gamma_{\mathrm{loc}, m}^{\nu^{\prime} v^{\prime \prime} \omega}\right]_{v^{\prime} v^{\prime \prime}}^{-1} \\
& \times \tilde{\chi}_{q}^{\nu^{\prime \prime}} G_{\mathrm{loc}, \nu+\omega} \Gamma_{m, \mathrm{loc}}^{\nu^{\prime \prime} v \omega}
\end{aligned}
$$

in turn contains the very same ladder but differs by a Green's function $G_{\text {loc }}$ in place of $\widetilde{G}_{k}$. The contribution $\Sigma_{1, k}^{(2)}=\Sigma_{\mathrm{d}, k}^{(2)}$, which is the same as the DF second-order diagram in Eq. (11), has to be subtracted in Eq. (28) to avoid a double counting of the second-order diagram (in $\Gamma_{\text {loc }}$ ) in the ladder series. Note that the matrix inversions in Eqs. (28) and (29) are performed with respect to the fermionic Matsubara frequencies $v^{\prime}$ and $v^{\prime \prime}$ for each value of $q$ (i.e., for fixed $\omega$ and $\mathbf{q}$ ).
According to Eqs. (25), (26), and (27), the Dyson equation in the spinor formalism reads as

$$
\begin{aligned}
\mathbf{G}_{k}^{-1} & =\boldsymbol{G}_{k}^{-1}-\boldsymbol{\Sigma}_{k} \\
& =\left(\begin{array}{cc}
G_{k}^{-1}-\Sigma_{1, k}-\Sigma_{2, k} & G_{k}^{-1}-\Sigma_{1, k}-\Sigma_{2, k} \\
G_{k}^{-1}-\Sigma_{1, k}-\Sigma_{2, k} & G_{k}^{-1}-G_{\mathrm{loc}, v}^{-1}-\Sigma_{1, k}
\end{array}\right) .
\end{aligned}
$$

Inverting (30) and performing the summation of the components of the obtained matrix [see Eq. (23)] we obtain the simple result

$$
\Sigma_{1 \mathrm{PI}, k}=\Sigma_{\mathrm{loc}, v}+\Sigma_{1, k}+\Sigma_{2, k} .
$$

Expanding the result Eq. (31) to leading order in $\widetilde{G}=G-$ $G_{\text {loc }}, \Sigma_{2}$ yields zero, while $\Sigma_{1}$ allows us to derive Eq. (11).

From Eq. (31) one can see, that the 1PI approach yields no spurious denominator for the lattice self-energy. Note that in the dual fermion approach, ${ }^{11}$ with the usual restriction to the two-particle local vertex, only the contribution $\Sigma_{1}$ [with the corresponding denominator, given in the Eq. (14)] appears, while $\Sigma_{2}$ corresponds to the contributions stemming from the three-particle local (one-particle reducible) vertex, see the discussion in Ref. 23.

At the same time, both contributions appear on the same ground in the 1PI approach already at the two-particle vertex level. As it is shown below, in Sec. V, the contribution $\Sigma_{2, k}$ yields however an enhanced asymptotics of the self-energy at large frequencies $v$. Therefore, at least the high-energy part of $\Sigma_{2, k}$ has to be compensated by the nonladder diagrams. In this respect, the situation in the 1PI approach is similar to the ladder approximation within the DГA approximation, where $\lambda$ corrections are needed to obtain the correct asymptotics of the self-energy. 
(a)

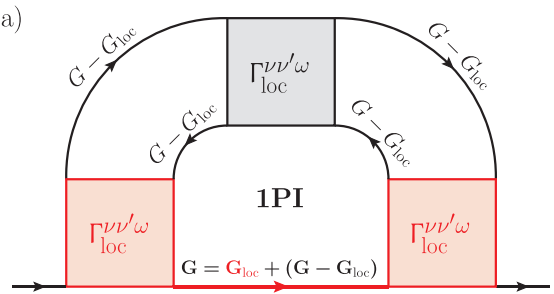

(b)

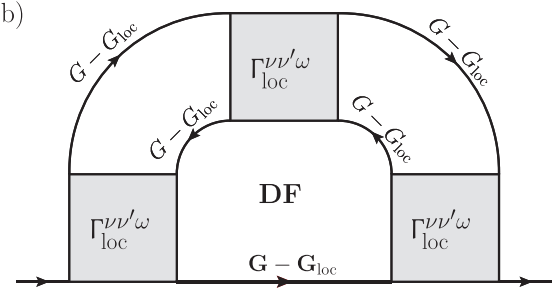

(c)

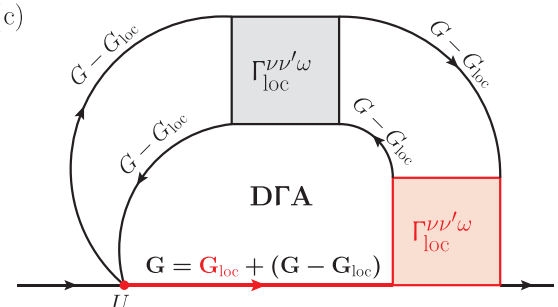

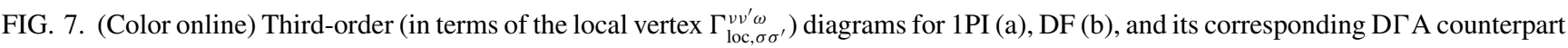
(c). The contribution of (a part of) the one-particle reducible three-particle vertex is marked in red in the 1PI and DГA diagrams.

\section{A. Comparison to the ladder DГA}

To compare the result (31) to the ladder DГA, let us represent the reducible local vertex via the irreducible one in a certain particle-hole channel

$$
\Gamma_{\mathrm{ir}, s(c)}^{\nu v^{\prime} \omega}=\left[\left(\Gamma_{\mathrm{loc}, s(c)}^{\nu v^{\prime} \omega}\right)_{\nu v^{\prime}}^{-1}+\chi_{\mathrm{loc}}^{0, v \omega} \delta_{\nu v^{\prime}}\right]^{-1}
$$

We now introduce the vertex

$$
\begin{aligned}
\Gamma_{\mathbf{q}, s(c)}^{\nu v^{\prime} \omega} & =\left[\left(\Gamma_{\mathrm{ir}, s(c)}^{\nu v^{\prime} \omega}\right)^{-1}-\bar{\chi}_{q}^{v} \delta_{\nu v^{\prime}}\right]^{-1}, \\
\bar{\chi}_{q}^{v} & =-\frac{1}{\beta} \sum_{\mathbf{k}} G_{k} G_{k+q}=\chi_{\mathrm{loc}}^{0, v \omega}+\tilde{\chi}_{q}^{v},
\end{aligned}
$$

where the inversion is performed with respect to the fermionic Matsubara frequencies $v$ and $v^{\prime}$. This way, after some algebraic manipulations we obtain

$$
\begin{aligned}
\Sigma_{1, k}= & \frac{1}{\beta} \sum_{\nu^{\prime} q} \sum_{m=c, s} A_{m} \Gamma_{\mathbf{q}, m}^{v v^{\prime} \omega} \bar{\chi}_{q}^{v^{\prime}}\left(G_{k+q}-G_{\mathrm{loc}, v+\omega}\right) \Gamma_{\mathrm{ir}, m}^{\nu^{\prime} v \omega} \\
& -\Sigma_{1, k}^{(2)}, \\
\Sigma_{2, k}= & \frac{1}{\beta} \sum_{v^{\prime} q} \sum_{m=c, s} A_{m}\left(\Gamma_{\mathbf{q}, m}^{\nu v^{\prime} \omega} \bar{\chi}_{q}^{v^{\prime}}-\Gamma_{\mathrm{loc}, m}^{\nu v^{\prime} \omega} \chi_{\mathrm{loc}}^{0, v^{\prime} \omega}\right) \\
& \times G_{\mathrm{loc}, v+\omega} \Gamma_{\mathrm{ir}, m}^{v^{\prime} v \omega} .
\end{aligned}
$$

In total this yields

$$
\begin{aligned}
\Sigma_{1 \mathrm{PI}, k}= & \Sigma_{\mathrm{loc}, v}+\frac{1}{\beta} \sum_{\nu^{\prime} q} \sum_{m=c, s} A_{m}\left(\Gamma_{\mathbf{q}, m}^{\nu \nu^{\prime} \omega} \bar{\chi}_{q}^{\nu^{\prime}}-\Gamma_{\mathrm{loc}, m}^{\nu \nu^{\prime} \omega} \chi_{\mathrm{loc}}^{0, v^{\prime} \omega}\right) \\
& \times \Gamma_{\mathrm{ir}, m}^{\nu^{\prime} v \omega} G_{k+q}-\Sigma_{1, k}^{(2)} .
\end{aligned}
$$

This result can be compared to the nonlocal self-energy in DГA as obtained previously in Ref. 9,

$$
\begin{aligned}
\Sigma_{\mathrm{D} \Gamma \mathrm{A}, k}= & \frac{1}{2} U n+\frac{U}{\beta} \sum_{\nu^{\prime} q} \bar{\chi}_{q}^{v^{\prime}}\left(A_{s} \Gamma_{\mathbf{q}, s}^{\nu v^{\prime} \omega}-A_{c} \Gamma_{\mathbf{q}, c}^{\nu v^{\prime} \omega}\right. \\
& \left.+\frac{1}{2} \Gamma_{\mathrm{loc}, c}^{\nu v^{\prime} \omega}-\frac{1}{2} \Gamma_{\mathrm{loc}, s}^{v v^{\prime} \omega}\right) G_{k+q} .
\end{aligned}
$$

From the comparison of the above expression to the 1PI ladder self-energy, Eq. (36), we can recognize an important difference: the bare interaction $U$ in Eq. (37) is replaced by the local particle-hole irreducible vertex $\Gamma_{\text {ir }}$ in Eq. (36), which is discussed diagrammatically in the next subsection.

\section{B. Differences in the 1PI, DF and DF A diagrammatics}

The different diagrammatic content of the ladder 1PI, ladder DF, and ladder DГA approaches is readily individuated by a direct inspection of the corresponding diagrams. We will start by considering a typical third-order diagram of the 1PI ladder series, shown in Fig. 7(a). Comparing to the corresponding diagram of the DF approach [Fig. 7(b)], it is evident that the latter does not include the term where the fermionic line at the bottom [bold red line in Fig. 7(a)] corresponds to a local Green's function. This is due to the fact that in the dual fermion space the propagation occurs via purely nonlocal Green's functions $G-G_{\text {loc }}$. Hence, when only the two-particle local vertex is considered as interaction among the dual fermions, there is no way to generate local Green's functions in the DF ladder diagrams. The difference between the diagrams of Fig. 7(a) and Fig. 7(b) corresponds to the contribution of the three-particle vertex in the DF approach [red part in Fig. 7(a)].

As in 1PI and in contrast to DF, also the corresponding DГA diagram [Fig. 7(c)] contains the full Green's function $G=G_{\text {loc }}+\left(G-G_{\text {loc }}\right)$, which also yields mixed terms with $G-G_{\text {loc }}$ propagators in the ladder part of the diagram and one local $G_{\text {loc }}$ outside the ladder (bottom of the diagram). Again, as for the 1PI diagram, the part of Fig. 7(c) colored in red corresponds to the contribution of the three-particle vertex in the DF approach.

At the same time, one should emphasize that the DГA ladder diagrams, as those depicted in Fig. 7(c), evidently represent only a subset of the 1PI ladder diagrams. This can be easily understood from a comparison of Fig. 7(a) and Fig. 7(c): In the 1PI approach all vertices appearing in the diagrams are the dynamical ones $\left(\Gamma_{\text {loc }}\right)$, while in DГA one of the vertex functions is replaced by its lowest-order counterpart, i.e., the bare interaction $U$. On the other hand, the 1PI ladder diagrams themselves are in turn just a subset of the more general set of diagrams generated by employing the parquet equations for the DГA instead of the ladder approximation.

What does the formal difference between 1PI and DГA mean physically? As it is illustrated in Fig. 8, the extra diagrams of 1PI correspond to considering nonlocal corrections to the irreducible vertex in the selected channel [Eq. (32)], while in ladder DГA calculations perfect locality of this vertex is assumed. Obviously the assumption of locality of the irreducible spin- and charge-vertex does not hold for the full DГA where nonlocal corrections to these vertices are generated via the self-consistent solution of the parquet equations. Hence, while, in general, the inclusion of a larger number of diagrams does not guarantee an improvement of 


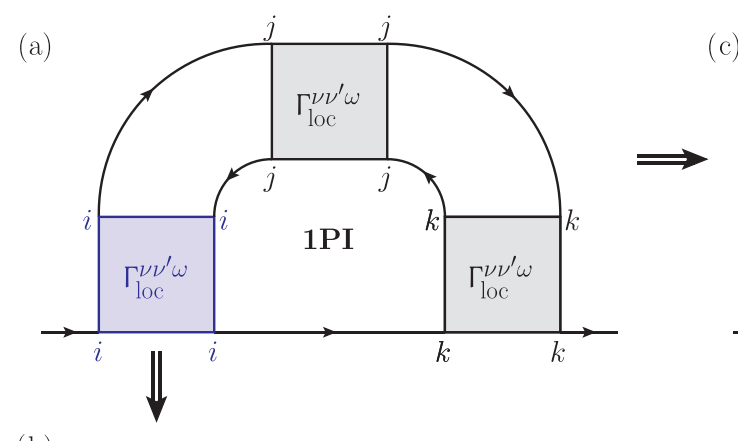

(b)

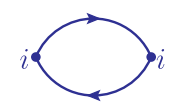

(d)

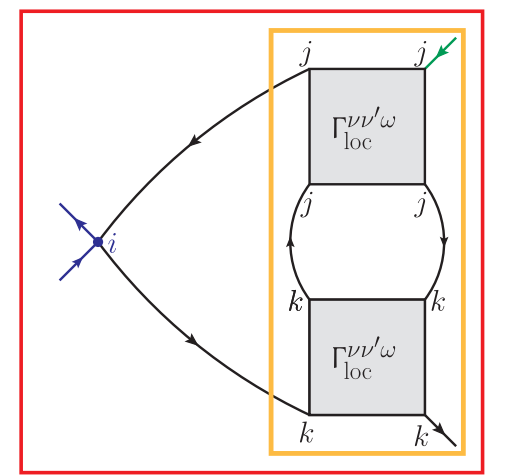

FIG. 8. (Color online) Expressing a 1PI diagram in terms of DГA where there is only a bare $U$ at the left-hand side of the diagram: We start from the specific 1PI diagram (a) and consider the specific contribution to the leftmost vertex shown in (b), so that (a) becomes (c). DГA, on the other hand, requires a bare $U$ on the leftmost side [see Fig. 7(c)]. Hence, the entire red box has to be interpreted as a DГA generated reducible vertex. The red box can be deformed to (d). The yellow box in diagram (d) clearly contains nonlocal contributions to the vertex irreducible in the longitudinal channel. To generate these in the DГA the full parquet treatment would be necessary.

a given approximation, in our case the additional nonlocal corrections for the irreducible (spin and charge) vertices are physically justifiable through the comparison with the full (parquet) DГA approach.

In order to demonstrate the differences between 1PI and DГA also analytically in the most transparent way, we can expand the DГA ladder expression for the self-energy [Eq. (37)] by representing $\bar{\chi}_{q}^{v^{\prime}}$ as a sum of local and nonlocal parts, $\chi_{\text {loc }}^{0, v^{\prime} \omega}+\tilde{\chi}_{q}^{v^{\prime}}$. Expanding to first order in $\tilde{\chi}_{q}^{v^{\prime}}$, we obtain

$$
\begin{aligned}
\Sigma_{\mathrm{D} \Gamma \mathrm{A}, k}^{(2)}= & \Sigma_{\mathrm{loc}, v}+\frac{1}{\beta} \sum_{\nu^{\prime} v^{\prime \prime} q}\left[A_{s} \Gamma_{\mathrm{loc}, s}^{\nu v^{\prime \prime} \omega} \widetilde{\chi}_{q}^{v^{\prime \prime}}\left(\bar{\Gamma}_{\mathrm{loc}, s}^{v^{\prime \prime} v^{\prime} \omega}-\frac{U}{2} \delta_{v^{\prime} v^{\prime \prime}}\right)\right. \\
& \left.+A_{c} \Gamma_{\mathrm{loc}, c}^{\nu v^{\prime \prime} \omega} \widetilde{\chi}_{q}^{\nu^{\prime \prime}}\left(\bar{\Gamma}_{\mathrm{loc}, c}^{\nu^{\prime \prime} v^{\prime} \omega}+\frac{U}{2} \delta_{\nu^{\prime} v^{\prime \prime}}\right)\right] \widetilde{G}_{k+q}
\end{aligned}
$$

where

$$
\begin{aligned}
\bar{\Gamma}_{\operatorname{loc}, s(c)}^{v v^{\prime} \omega} & = \pm U\left[\delta_{\nu v^{\prime}}-\Gamma_{\mathrm{ir}, s(c)}^{\nu v^{\prime} \omega} \chi_{0 \omega, \mathrm{loc}}^{\nu}\right]^{-1} \\
& = \pm U \sum_{v^{\prime \prime}} \Gamma_{\operatorname{loc}, s(c),\left[\Gamma_{\mathrm{ir}, s(c)}^{\omega}\right]_{v^{\prime \prime} \nu^{\prime}}^{-1}}^{-1}
\end{aligned}
$$

Expanding the corresponding expression for the 1PI selfenergy in Eq. (36) in a similar manner, one obtains $\Sigma_{1 \mathrm{PI}, k}^{(2)}=$ $\Sigma_{\text {loc, },}+\Sigma_{\mathrm{d}, k}^{(2)}$. Comparing this result to the corresponding DГА one [Eq. (38)] one observes two differences: (i) The factor $1 / 2$ in Eq. (11), which avoids double counting of diagrams is replaced by an explicit subtraction of double counting terms $\pm U / 2$ in Eq. (38) for the $\mathrm{D \Gamma A}$. The reason for this is the asymmetric form of the DГA self-energy correction compared to the 1PI one (bare $U$ in DГA vs the full vertex on in 1PI on the left-hand side of the self-energy diagrams, see Fig. 7). (ii) The second, more important, difference between the two expressions is that $\bar{\Gamma}_{\mathrm{loc}, s(c)}^{\nu v^{\prime} \omega}$ in Eq. (38) is replaced by $\Gamma_{\mathrm{loc}, s(c)}^{\nu v^{\prime} \omega}$ in $\Sigma_{1 \mathrm{PI}}^{(2)}$. Hence, the difference between $\bar{\Gamma}_{\operatorname{loc}, s(c)}^{v \nu^{\prime} \omega}$ and $\Gamma_{\operatorname{loc}, s(c)}^{v v^{\prime} \omega}$ marks a particular set of nonlocal corrections to the self-energy, naturally generated in the 1PI ladder diagrams, but neglected in the ladder expansions of the DГА.

The interpretation of the ladder 1PI expression derived in this section can be summarized as follows: in the ladder approximation, the 1PI diagrams include terms not present in DГ A and DF. In the latter approaches, these are generated by going beyond the ladder approximation to $\mathrm{D} \Gamma \mathrm{A}$ and beyond the two-particle vertex in DF, respectively. The numerical effort of performing a ladder 1PI calculation is much smaller compared to the full (parquet-based) DГA, or to the DF with the three-particle vertex. In a sense the 1PI approach better utilizes the information contained in the single-particle Green's function and two-particle vertex.

\section{NUMERICAL RESULTS}

In this section, we present numerical results for nonlocal corrections to the self-energy of the two-dimensional Hubbard model obtained by means of the ladder 1PI formalism and compare them with the corresponding DF and DГA results. We consider the relevant case of the Hubbard model on a 

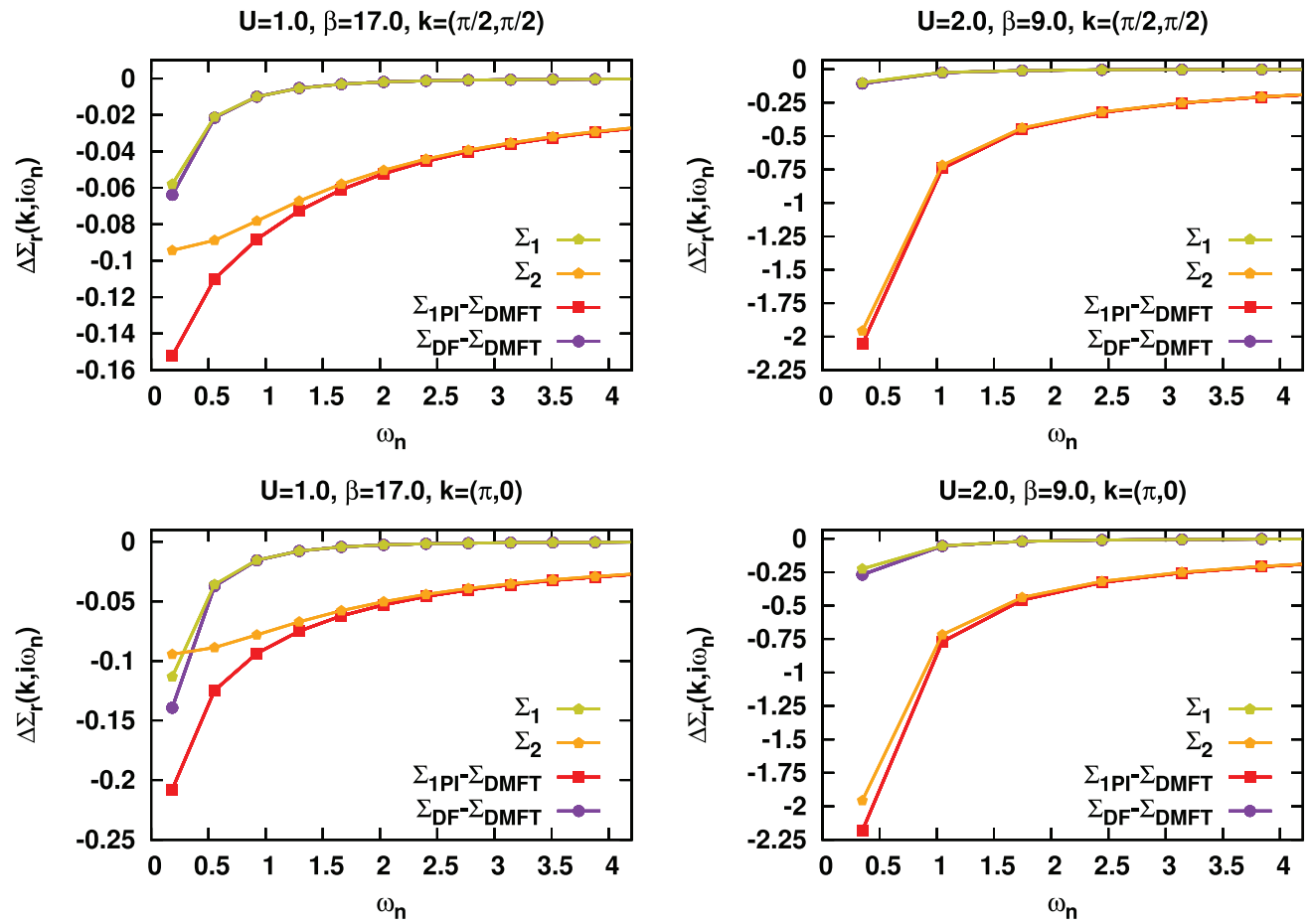

FIG. 9. (Color online) Nonlocal corrections $\Delta \Sigma_{\mathrm{r}}\left(\mathbf{k}, i \omega_{n}\right)=\Sigma_{\mathrm{r}}\left(\mathbf{k}, i \omega_{n}\right)-\Sigma_{\text {loc }}\left(i \omega_{n}\right)(r=1 \mathrm{PI}$ [Eq. (31)] and DF [Eq. (14)], respectively) to the DMFT (local) self-energy for the $d=2$ Hubbard model on a square-lattice at half filling for two different values of $U$, two different $\mathbf{k}$ points on the Fermi surface [i.e., $\mathbf{k}=\left(\frac{\pi}{2}, \frac{\pi}{2}\right)$, nodal point, and $\mathbf{k}=(\pi, 0)$, antinodal point], and temperatures slightly above the corresponding $T_{\mathrm{N}}$ of DMFT. For the 1PI results the single contributions $\Sigma_{1}$ [Eq. (28)] and $\Sigma_{2}$ [Eq. (29)] are also shown separately. Note, all self energies are purely imaginary; this imaginary part is shown.

(two-dimensional) square lattice with nearest-neighbor hopping $t$ at half-filling, where the effect of nonlocal correlations beyond DMFT is expected to be particularly strong. Note that in the following all energy scales, such as the Hubbard interaction parameter $U$ and the temperature $T=1 / \beta$, will be given in units of the half bandwidth $W / 2=4 t=1$. Furthermore, one should bear in mind that for the half-filled Hubbard model the self-energy evaluated for $\mathbf{k}$ points at the Fermi surface is purely imaginary as a function of Matsubara frequencies (besides the constant Hartree contribution $\frac{U n}{2}$ ). Hence, in order to keep the notation as simple as possible, $\Sigma$ refers to the imaginary part of the self-energy, i.e., $\Sigma \widehat{=} \operatorname{Im} \Sigma$, in the sections below.

Before presenting our numerical results in the next two subsections, let us stress that the only possibility to perform a one-by-one comparison between the diagrammatic methods stands for the (non-self-consistent) one-shot calculations. As discussed in Sec. IV, only in this case the exact relations between the three different approaches and their diagrammatic content can be identified. Hence, this analysis is performed first. The obtained results do not necessarily represent the final, physical results of the three methods. In a separate subsection, we therefore look at the trends emerging when going beyond the one-shot calculations. We note that because of the different ways the self-consistency is implemented (inner and outer self-consistency loop in $\mathrm{DF}^{28}$ Moriyaesque $\lambda$ correction $^{29}$ in DГA and 1PI), as well as the different possible levels of approximation (ladder or parquet diagrams) an identification of equivalent levels of approximation as in the one-shot case is not possible. Also for keeping the comparison among different methods as precise as possible, we present our numerical results on the Matsubara frequency axis only, avoiding the additional, and to some extent uncontrolled, effects of an analytic continuation.

\section{A. One-shot calculations}

In this subsection, we will focus on non-self-consistent oneshot calculations for nonlocal corrections to the (local) DMFT self-energy: this approach represents an expansion around DMFT, where the auxiliary local AIM [Eq. (2)] is not changed with respect to DMFT and the DMFT Green's functions [Eq. (4)] are not renormalized by a feedback of the nonlocal self-energy. As one can understand from the discussion in the previous sections, examining (non-self-consistent) one-shot calculations corresponds to considering well-defined sets of diagrams for the lattice electrons. This way we are able to individuate the general trends obtained by the three approaches (1PI, DF, and DГA) emerging purely from their different diagrammatic content.

For the sake of conciseness, we will mainly discuss the numerical results obtained with ladder calculations, since they are most frequently adopted in previous papers, ${ }^{12,17,22,30}$ and the inclusion of ladder diagrams proved to be essential to correctly describe crucial features of the two- and threedimensional physics. Examples are the pseudogap ${ }^{12}$ in $d=2$ or the critical exponents in $d=3$ dimensions. ${ }^{22}$

In Fig. 9 we present our results for one-shot calculations of the nonlocal corrections to the DMFT self-energy, $\Delta \Sigma_{\mathrm{r}}\left(\mathbf{k}, i \omega_{n}\right)=\Sigma_{\mathrm{r}}\left(\mathbf{k}, i \omega_{n}\right)-\Sigma_{\mathrm{loc}}\left(i \omega_{n}\right)$ for $r=1 \mathrm{PI}$ [Eq. (31)] and DF [Eq. (14)], respectively, on the Matsubara frequency 

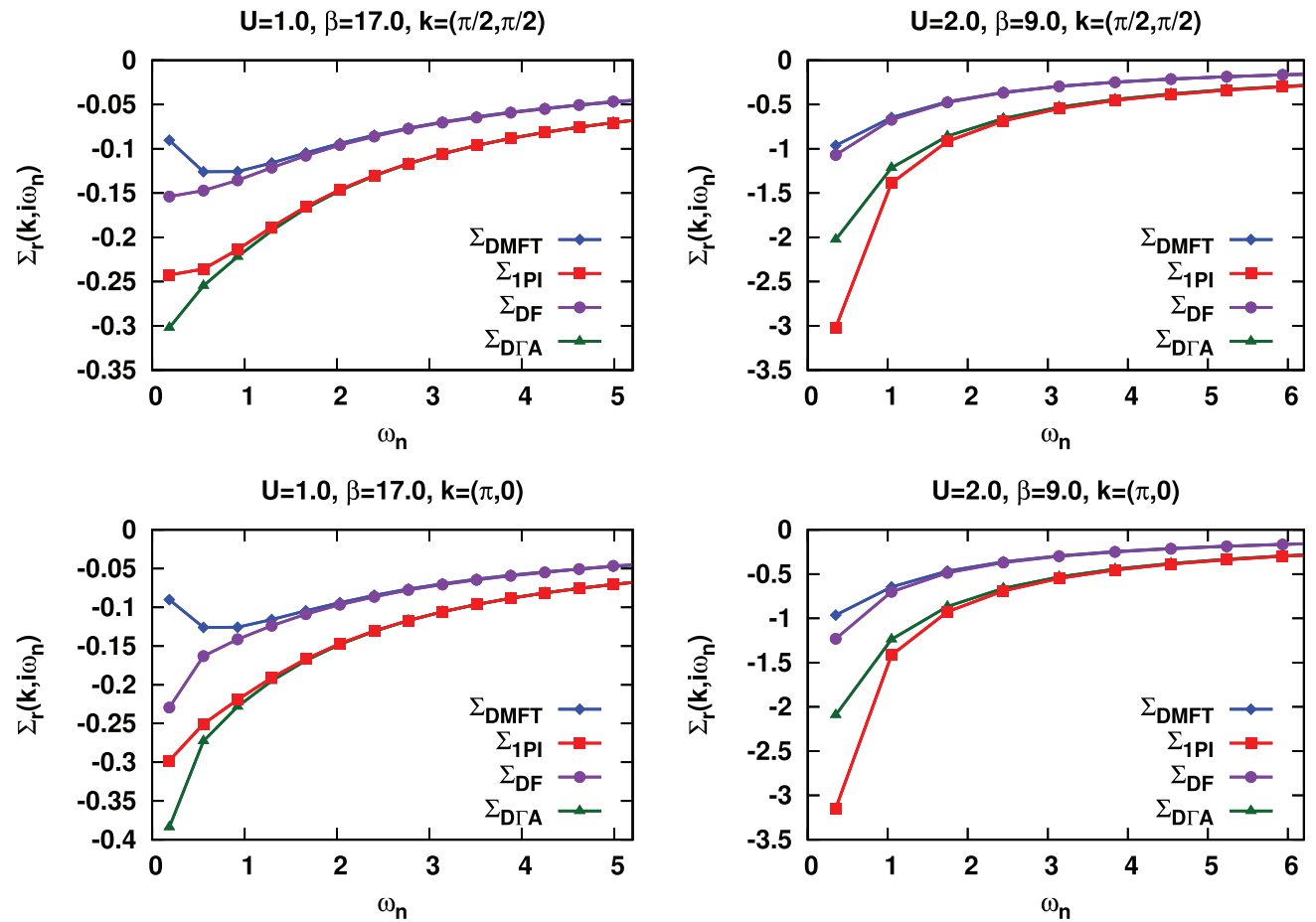

FIG. 10. (Color online) Self-energies (imaginary part) obtained with one-shot calculations for the 1PI approach vs DF, DГA, and DMFT self-energies for the same parameters as in Fig. 9.

axis. For the 1PI approach we also show its two contributions $\Sigma_{1}$ [Eq. (28)] and $\Sigma_{2}$ [Eq. (29)] separately. Note, that since no self-consistent adaption of the underlying local model is performed, the local self-energy coincides with the DMFT one, i.e., $\Sigma_{\mathrm{loc}}\left(i \omega_{n}\right)=\Sigma_{\mathrm{loc}}^{\mathrm{DMFT}}\left(i \omega_{n}\right)$. Data for weak $(U=1.0)$ and intermediate-to-strong coupling $(U=2.0)$ and for two different $\mathbf{k}$ points on the Fermi surface are presented. The temperature has been chosen to be slightly above the onset of the antiferromagnetic ordering (Néel temperature, $T_{\mathrm{N}}^{\mathrm{DMFT}}$ ) obtained in DMFT, aiming to maximize the effect of nonlocal correlations. One can see that, quite generally, the nonlocal corrections in the considered approaches increase the imaginary part of the self-energy, making its low-frequency dependence less metallic. Comparing the relative magnitudes of the nonlocal corrections shown in Fig. 9, the contribution of $\Sigma_{1}$ of the 1PI approach appears always rather small even though the $U$ and $T$ values have been selected very close to the antiferromagnetic instability of DMFT. The reason for this behavior is that in $\Sigma_{1}$ one has to perform $\mathbf{k}$ summations over terms containing $G-G_{\text {loc }}$, which yields small results since in a one-shot calculation, $\sum_{\mathbf{k}} G_{k}-G_{\text {loc }}=0$ because of the DMFT self consistency [Eq. (3)]. Let us also note that in one-shot calculations, the $\Sigma_{1}$ part of the 1PI correction [Eq. (28)] almost exactly coincides with the DF correction $\Sigma_{\mathrm{DF}}-\Sigma_{\mathrm{DMFT}}$, albeit without the denominator in Eq. (14). For the data presented here, the effect of the denominator is found to be rather small. On the contrary, in $\Sigma_{2}$ a mixing of local and nonlocal contributions occurs, because one single Green's function $G_{\text {loc }}$ enters instead of $G-G_{\text {loc }}$ [see Eq. (29)]. Hence this term becomes significantly larger than $\Sigma_{1}$.

However, as it was already mentioned in Sec. IV, the contribution $\Sigma_{2}$ displays an enhanced high-frequency asymp- totics, while $\Sigma_{1}$ decays faster than $\frac{1}{i \omega_{n}}$ and preserves the exact asymptotic behavior of the self-energy when added to the local self-energy of DMFT. The reason for this is again that $\Sigma_{1}$ is constructed from $G-G_{\text {loc }}$ only, which decays as $\frac{1}{\left(i \omega_{n}\right)^{2}} . \Sigma_{2}$ has an explicit $\frac{1}{i \omega_{n}}$ contribution from the $G_{\text {loc }}$ term, which leads to a (spurious) correction of the already exact $\frac{1}{i \omega_{n}}$ behavior of the DMFT self-energy. We note here that the enhanced asymptotic of $\Sigma_{2}$ and, hence, of the 1PI approach, is exactly the same as in $\mathrm{D} \Gamma \mathrm{A}^{31}$ as one can observe in Fig. 10. Similarly to the DГA case, the enhanced asymptotic is corrected either by treating the full parquet set of diagrams, or enforcing the condition $\sum_{\mathbf{q}} \chi(\mathbf{q})=\chi_{\text {AIM }}$ at the ladder level via Moriyaesque $\lambda$-corrections, ${ }^{17}$ see the results in the next subsection.

In Fig. 10 we plot the self-energy obtained from one-shot ladder calculations for $1 \mathrm{PI}, \mathrm{DF}$ and $\mathrm{D} \Gamma \mathrm{A}$ in comparison to DMFT. For 1PI and DГA, nonlocal corrections are large as expected from the proximity to the DMFT Néel temperature. In the weak-coupling regime (i.e., for $U=1.0$ ), one further observes that the 1PI correction is smaller than the corresponding ВГА correction. The reason for this is that the $U$ appearing in the $\mathrm{DГА}$ equation (37) is replaced by the irreducible vertex in the 1PI formula. At small values of the interaction parameter $U$, the (irreducible) vertex is smaller ${ }^{9,32}$ than the bare interaction due to metallic screening. Therefore, nonlocal corrections obtained within the 1PI formalism tend to be smaller than the one obtained in DГА.

The situation is completely reversed in the intermediate-tostrong coupling regime $(U=2.0)$. Here, the local (irreducible) vertex is strongly enhanced ${ }^{9,32,33}$ compared to the bare Hubbard interaction $U$, due to the formation of the local moment 

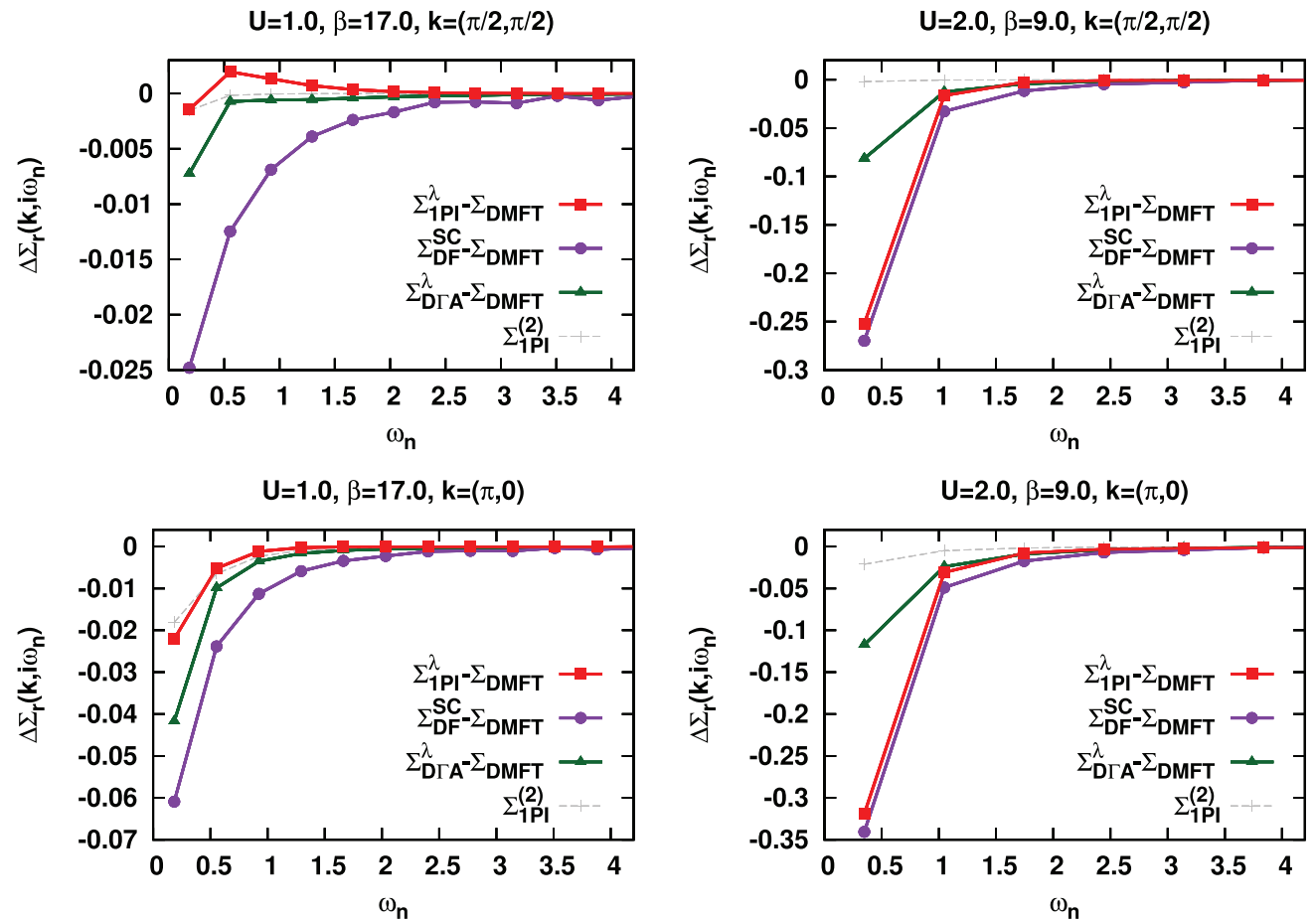

FIG. 11. (Color online) Nonlocal corrections $\Delta \Sigma_{\mathrm{r}}\left(\mathbf{k}, i \omega_{n}\right)=\Sigma_{\mathrm{r}}\left(\mathbf{k}, i \omega_{n}\right)-\Sigma_{\text {loc }}\left(i \omega_{n}\right)(r=1 \mathrm{PI}$, DF, and DГA, respectively) as in Fig. 9 , but for calculations including Moriyaesque $\lambda$ corrections (1PI and DГA) or self-consistency (DF). Besides the ladder 1PI results we also include the results from the second-order diagram $\Sigma_{1 \mathrm{PI}}^{(2)}\left(\mathbf{k}, i \omega_{n}\right)=\Sigma_{\mathrm{d}}^{(2)}\left(\mathbf{k}, i \omega_{n}\right)$ given in Eq. (11).

in the proximity of the Mott phase. Hence, the 1PI self-energy correction is larger than that obtained in DГA.

In the present implementation of 1PI and DГA the calculation of the Neel-temperature $T_{\mathrm{N}}$ by means of a $\lambda$ correction
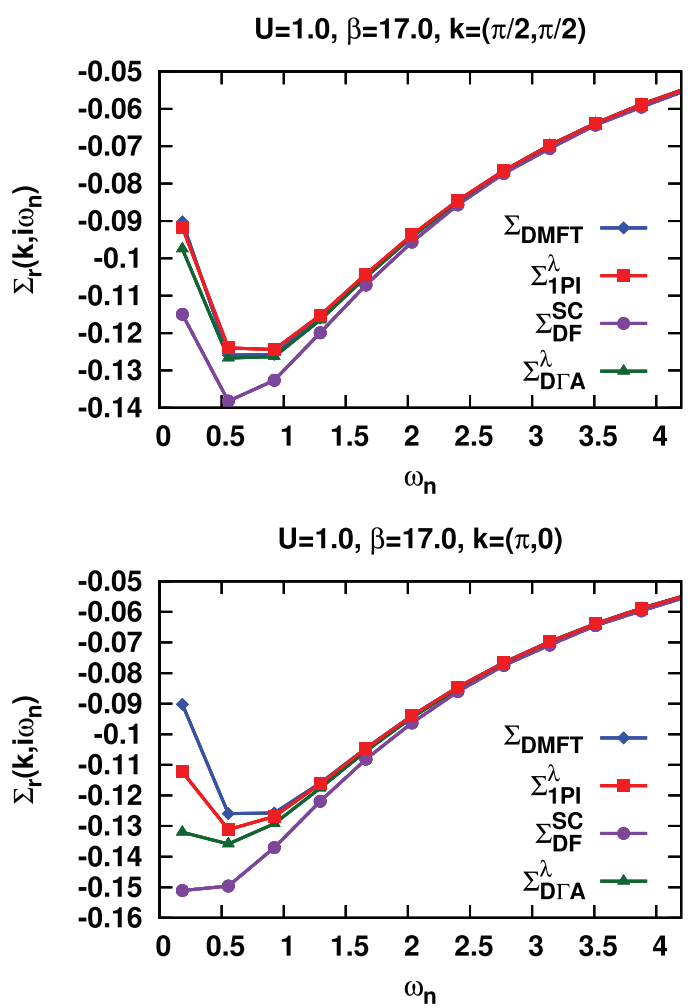

is purely based on the asymptotic behavior of the (nonlocal) self-energy. This is the same in both approaches and, hence, one would get the same transition temperatures. However, an improved scheme of $\lambda$ corrections or a self-consistent
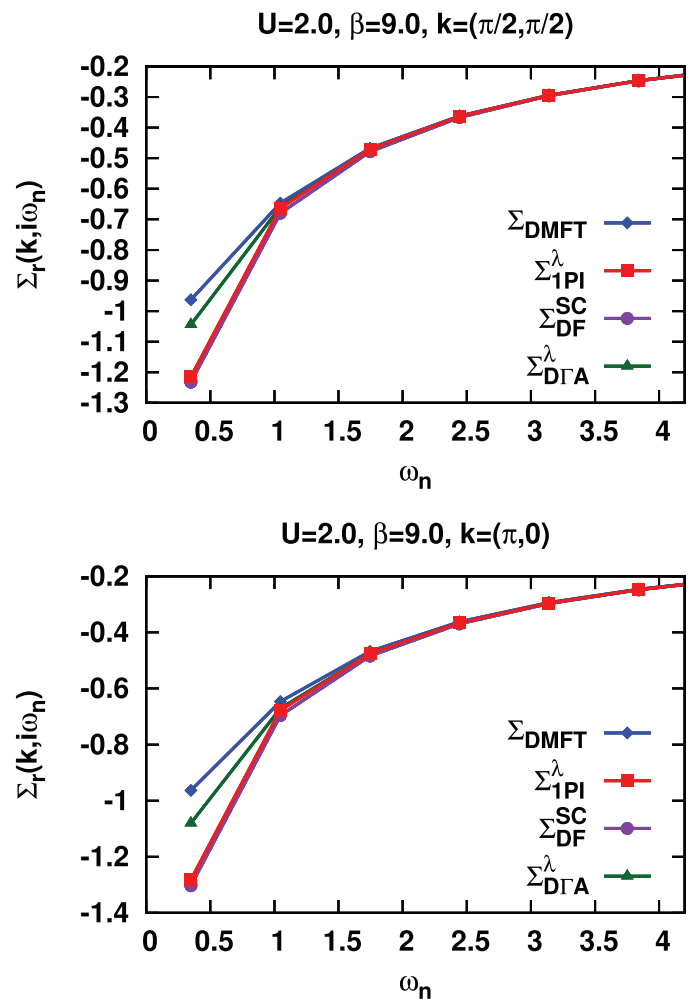

FIG. 12. (Color online) Self-energies obtained with the 1PI approach including $\lambda$-corrections vs self-consistent DF, $\lambda$ corrected DГA, and DMFT self-energies for the same parameters as in Fig. 11. 
treatment of these theories is expected to yield different $T_{\mathrm{N}}$ 's. In Ref. $22 T_{\mathrm{N}}$ was found smaller in DГA than the one estimated in $\mathrm{DCA}^{34}$ or in lattice quantum Monte Carlo ${ }^{35}$ at weak coupling, indicating a possible overestimation of the nonlocal correlation effects. As it was argued in Ref. 22, nonlocal corrections to the charge- and particle-particle irreducible channels, which can be rigorously included only by performing the DГA at the parquet level, might be responsible for this. Hence, the 1PI approach, which partly takes such corrections into account (see Fig. 8), is rather promising to improve the agreement between the diagrammatic and the cluster estimations of $T_{\mathrm{N}}$ in the Hubbard model, even in the (self-consistent) ladder approximation. This may also hold true in the strong-coupling regime, where $T_{\mathrm{N}}$ was slightly larger in ladder DГA than in the cluster methods.

As for the comparison with the DF self-energy one can see that it is smaller than the corresponding 1PI and DГA ones. The reason for this is the same as discussed for the contribution $\Sigma_{1}$ to the 1PI self-energy. However, one should consider, that the different ways of self-consistency for 1PI, DF and DГA can change this situation dramatically.

\section{B. Self-consistency and Moriyaesque $\lambda$ corrections}

The analysis of the one-shot results has shown the existence of a well-defined hierarchy in the relative magnitude of the nonlocal corrections. It is however expected that the overall size of the nonlocal corrections will be strongly modified by the inner and outer self-consistency loops in $\mathrm{DF}^{12}$ on the one hand and the inclusion of the Moriyaesque $\lambda$ corrections in $\mathrm{D} \Gamma \mathrm{A}^{17}$ and $1 \mathrm{PI}^{36}$ on the other. These effects are briefly analyzed in this subsection.

The results of the self-consistent DF, DГA, and 1PI approaches are presented in Fig. 11. Comparing them to Fig. 9, one observes that the inclusion of the $\lambda$ corrections in DГA and 1 PI (which reduces the value of $T_{\mathrm{N}}$ from the overestimated DMFT value ) leads to a significant reduction of the nonlocal corrections to the self-energy (note the different scales in the two figures). This has been observed previously for DГA. ${ }^{17,22}$ Hence, the $\lambda$-corrected results become much more similar to those obtained in self-consistent DF calculations. In particular, at strong coupling, 1PI and DF agree rather well. The previously mentioned hierarchy in the relative magnitude of the nonlocal corrections to DMFT of 1PI and DГA results is fully preserved by the Moriyaesque $\lambda$ corrections (see Fig. 11): At weak coupling $(U=1.0)$ the 1PI corrections remain smaller than the DГA ones due to the metallic screening of the irreducible vertex, while for stronger couplings $(U=2.0)$ the enhancement of the same vertex due to the vicinity of the MIT leads to larger corrections for the 1PI approach with respect to the DГА. Note that the small value of the nonlocal part of the self-energy in the 1PI approach at $U=1.0$ (especially in the nodal direction) may result from a simplified way of considering self-consistent effects through the $\lambda$ correction. Since this correction is determined solely from the asymptotic behavior of the self-energy at large frequencies, it may yield an overestimation of the effect of nonladder diagrams in the 1PI approach in the low-frequency region.

In Fig. 12 we present the corresponding results for the self-energies. For $U=1.0$ one can see, that at the consid- ered temperature one observes metallic behavior in all the approaches, except for the DF data in the $(\pi, 0)$ direction. We have verified, however, that even for this relatively small value of $U$ the nonlocal 1PI corrections, though smaller than the DГ A and DF ones, eventually overcome the metallic behavior of the DMFT self-energy at sufficiently small temperatures, consistent with the results of Ref. 38. We emphasize that, for $U=1.0$, cluster extensions of DMFT would predict, instead, a low-temperature metallic phase. ${ }^{39}$ This confirms the necessity of including long-range antiferromagnetic fluctuations beyond DMFT in order to capture correctly the interplay of the Mott-Hubbard transition and antiferromagnetism (at $T=0$ ), whose nature gradually changes from Slater to Heisenberg. ${ }^{40,41}$

\section{CONCLUSIONS}

In this paper, we have developed a one-particle irreducible (1PI) approach for including nonlocal spatial correlations on top of the local correlations of dynamical mean-field theory. We have compared it with the existing state-of-the-art diagrammatic extensions of DMFT, namely dual fermion (DF) and dynamical vertex approximation (DГA).

The starting point of the 1PI approach is the generating functional formalism in the functional integral representation. Similar to the DF theory, we decouple local and nonlocal degrees of freedom by means of a Hubbard-Stratonovich transformation and integrate out the local degrees of freedom. However, instead of expanding the logarithm of the local generating functional in the source fields, which would lead to local one-particle reducible two- and more-particle vertex functions, we pass on to the 1PI local functional by means of a Legendre transform. For the sake of conciseness, we have considered in this work the two typical approximations for the diagrammatic methods: (i) the restriction to the local two-particle vertices and (ii) the ladder approximation for the self-energy. With these assumptions, we could show how the ladder self-energy diagrams generated by the 1PI approach also include contributions from local one-particle reducible three-particle vertices, which, in the DF approach, can only be generated when explicitly computing the local three-particle vertex. Hence, when adopting the usual approximations, the 1PI approach contains a larger set of diagrams than DF.

Let us also stress that the 1PI approach prevents the generation of spurious reducible diagrams present in the DF self-energy when restricting oneself to the two-particle vertices. $^{23}$ In this respect, the 1PI approach can be further used for a consistent formulation of the renormalization of the DF approach, restricted to the two-particle level (e.g., within the functional renormalization-group analysis). ${ }^{42}$

As for the comparison with the DГA, we note that its derivation is purely based on diagrammatic considerations rather than on a path-integral formulation. However, when comparing the diagrams defining the ladder approximation of DГA with the corresponding ones from the 1PI approach, it turns out that they have a similar structure. We observe that-as for the DF approach-the 1PI method allows us to 
treat diagrams which are neglected in the ladder DГ A analysis, but are present in its parquet generalizations.

Beyond a detailed analysis of the diagrammatics of the 1PI, $\mathrm{DF}$, and $\mathrm{D} \Gamma \mathrm{A}$, we have also compared the numerical results of the three approaches. For non-self-consistent calculations DF yields substantially weaker corrections to DMFT than DГA and 1PI. Self-consistent results, which in the case of 1PI and DГA are mimicked by a Moriyaesque $\lambda$ correction, are more similar. Here, we observe the general trend that 1PI yields somewhat stronger corrections to the DMFT self-energy than DГA at intermediate-to-strong coupling, yielding results, which are close to those in the DF approach. At weak coupling we find the nonlocal corrections to the self-energy in the 1PI approach to be smaller, than those in the DГA and DF approaches. We trace this back to the additional Feynman diagrams of 1PI which, in comparison to DГA, substitute a bare interaction $U$ by a local vertex. At weak coupling, this local vertex is smaller than $U$ because of metallic screening processes. At strong coupling it is larger, because of the formation of a local moment associated to strong spin fluctuations at the MIT.

In summary, the 1PI approach unifies features of the DF and DГA approaches. Restricting ourselves to (i) a truncation of the approaches at the two-particle local vertex level and (ii) ladder diagrams generated from these, the 1PI allows for a treatment of the nonlocal self-energy effects, accounting for the nonladder scattering processes.

\section{ACKNOWLEDGMENTS}

We thank A. I. Lichtenstein for critical comments on the manuscript. We gratefully acknowledge financial support through the Austrian-Russian joint project from the Austrian Science Fund (FWF), Project No. I-610-N16 (GR, AT) and Russian Fund of Basic Research (RFBR), Grant No. 1002-91003-ANFa (AK); RFBR grant 13-02-00050 (VA); the Program of the Russian Academy of Science Presidium Quantum microphysics of condensed matter 12-P-2-1017, 12-M-23-2020 and the grant of the Ministry of education and science of Russia No. 14.18.21.0076 (VA); as well as the European Research Council under the European Union's Seventh Framework Programme (FP/2007-2013)/ERC through Grant Agreement No. 306447 (KH). The numerical calculations presented in this paper have been performed on the Vienna Scientific Cluster (VSC).

\section{APPENDIX: 1PI TRANSFORMATIONS OF THE GENERATING FUNCTIONAL}

\section{Calculation of $J\left[\phi^{+}, \phi\right]$}

In order to pass to the 1PI functional, we change variables of integration $\widetilde{c}^{+}, \widetilde{c}$ to the Legendre transformed quantities $\phi^{+}, \phi$ [see Eq. (18) and the definition of $\tilde{\eta}$ below]:

$$
\widetilde{c}_{k \sigma}^{+}=-\frac{\delta \Gamma_{\mathrm{DMFT}}}{\delta \phi_{k \sigma}}-\eta_{k \sigma}^{+} \quad \widetilde{c}_{k \sigma}=\frac{\delta \Gamma_{\mathrm{DMFT}}}{\delta \phi_{k \sigma}^{+}}-\eta_{k \sigma} .
$$

Considering that the source fields $\eta^{+}$and $\eta$ do not depend on $\widetilde{c}^{+}$and $\widetilde{c}$, the corresponding matrix $M\left[\phi^{+}, \phi\right]$ of this transformation can be written as

$$
d\left(\begin{array}{c}
\widetilde{c}_{k \sigma}^{+} \\
\widetilde{c}_{k \sigma}
\end{array}\right)=\underbrace{\left(\begin{array}{cc}
-\frac{\delta^{2} \Gamma_{\mathrm{DMFT}}}{\delta \phi_{k^{\prime} \sigma^{\prime}}^{+} \delta \phi_{k \sigma}} & -\frac{\delta^{2} \Gamma_{\mathrm{DMFT}}}{\delta \phi_{k^{\prime} \sigma^{\prime}} \delta \phi_{k \sigma}} \\
\frac{\delta^{2} \Gamma_{\mathrm{DMFT}}}{\delta \phi_{k^{\prime} \sigma^{\prime}}^{+} \delta \phi_{k \sigma}^{+}} & \frac{\delta^{2} \Gamma_{\mathrm{DMFT}}}{\delta \phi_{k^{\prime} \sigma^{\prime}} \delta \phi_{k \sigma}^{+}}
\end{array}\right)}_{M\left[\phi^{+}, \phi\right]} d\left(\begin{array}{c}
\phi_{k^{\prime} \sigma^{\prime}}^{+} \\
\phi_{k^{\prime} \sigma^{\prime}}^{+}
\end{array}\right) .
$$

The calculation of the first (which will be needed later) and the second functional derivatives of the functional $\Gamma_{\mathrm{DMFT}}\left[\phi^{+}, \phi\right]$ with respect to the fields $\phi^{+}$and $\phi$ can be performed straightforwardly using the explicit expression for $\Gamma_{\text {DMFT }}$ given in Eq. (21). The results are

$$
\begin{gathered}
\frac{\delta \Gamma_{\mathrm{DMFT}}\left[\phi^{+}, \phi\right]}{\delta \phi_{k \sigma}}=\frac{1}{\beta} G_{\mathrm{loc}, \nu}^{-1} \phi_{k \sigma}^{+}-\frac{1}{\beta^{3}} \sum_{k_{1} q} \sum_{\sigma_{1}} \widetilde{\Gamma}_{\mathrm{loc}, \sigma \sigma_{1}}^{v_{1} v \omega} \\
\frac{\delta \phi_{k+q, \sigma}^{+} \phi_{k_{1} \sigma_{1}}^{+} \phi_{k_{1}+q, \sigma_{1}},}{\frac{\delta \Gamma_{\mathrm{DMFT}}\left[\phi^{+}, \phi\right]}{\delta \phi_{k \sigma}^{+}}=}-\frac{1}{\beta} G_{\mathrm{loc}, \nu}^{-1} \phi_{k \sigma}+\frac{1}{\beta^{3}} \sum_{k_{1} q} \sum_{\sigma_{1}} \widetilde{\Gamma}_{\mathrm{loc}, \sigma \sigma_{1}}^{\nu v_{1} \omega} \phi_{k+q, \sigma} \\
\times \phi_{k_{1}+q, \sigma_{1}}^{+} \phi_{k_{1} \sigma_{1}},
\end{gathered}
$$

for the first derivatives and

$$
\begin{aligned}
& \frac{\delta^{2} \Gamma_{\mathrm{DMFT}}}{\delta \phi_{k^{\prime} \sigma^{\prime}} \delta \phi_{k \sigma}^{+}}=-\frac{1}{\beta} G_{\mathrm{loc}, v}^{-1} \delta_{k k^{\prime}} \delta_{\sigma \sigma^{\prime}} \\
& -\frac{1}{\beta^{3}} \sum_{q} \widetilde{\Gamma}_{\mathrm{loc}, \sigma \sigma^{\prime}}^{\nu v^{\prime} \omega} \phi_{k^{\prime}+q, \sigma^{\prime}}^{+} \phi_{k+q, \sigma} \\
& +\frac{\delta_{\sigma \sigma^{\prime}}}{\beta^{3}} \sum_{q, \sigma_{1}} \widetilde{\Gamma}_{\text {loc, }, \sigma \sigma_{1}}^{v, v+\omega, \nu^{\prime}-v} \phi_{k^{\prime}+q, \sigma_{1}}^{+} \phi_{k+q, \sigma_{1}} \\
& \frac{\delta^{2} \Gamma_{\mathrm{DMFT}}}{\delta \phi_{k^{\prime} \sigma^{\prime}}^{+} \delta \phi_{k \sigma}^{+}}=-\frac{1}{\beta^{3}} \sum_{q} \widetilde{\Gamma}_{\mathrm{loc}, \sigma \sigma^{\prime}}^{v, \nu^{\prime}-\omega} \phi_{k+q, \sigma} \phi_{k^{\prime}-q, \sigma^{\prime}} \\
& \frac{\delta^{2} \Gamma_{\mathrm{DMFT}}}{\delta \phi_{k^{\prime} \sigma^{\prime}} \delta \phi_{k \sigma}}=\frac{1}{\beta^{3}} \sum_{q} \widetilde{\Gamma}_{\mathrm{loc}, \sigma \sigma^{\prime}}^{v^{\prime}-\omega, v \omega} \phi_{k^{\prime}-q, \sigma^{\prime}}^{+} \phi_{k+q, \sigma}^{+} \\
& \frac{\delta^{2} \Gamma_{\mathrm{DMFT}}}{\delta \phi_{k^{\prime} \sigma^{\prime}}^{+} \delta \phi_{k \sigma}}=\frac{1}{\beta} G_{\mathrm{loc}, v}^{-1} \delta_{k k^{\prime}} \delta_{\sigma \sigma^{\prime}} \\
& +\frac{1}{\beta^{3}} \sum_{q} \widetilde{\Gamma}_{\mathrm{loc}, \sigma \sigma^{\prime}}^{v \nu^{\prime} \omega} \phi_{k+q, \sigma}^{+} \phi_{k^{\prime}+q, \sigma^{\prime}} \\
& -\frac{\delta_{\sigma \sigma^{\prime}}}{\beta^{3}} \sum_{q, \sigma_{1}} \widetilde{\Gamma}_{\mathrm{loc}, \sigma \sigma_{1}}^{v+\omega, v^{\prime}-v} \phi_{k+q, \sigma_{1}}^{+} \phi_{k^{\prime}+q, \sigma_{1}} .
\end{aligned}
$$

for the second functional derivatives. $\widetilde{\Gamma}_{\mathrm{loc}, \sigma \sigma^{\prime}}^{\nu v^{\prime} \omega}$ is defined below Eq. (21).

Next, we single out the factor $\left[\beta G_{\mathrm{loc}, \nu}\right]^{-1}$ from the Jacobian $M\left[\phi^{+}, \phi\right]=\left[\beta G_{\mathrm{loc}, v}\right]^{-1} \tilde{M}\left[\phi^{+}, \phi\right]$ and omit it since it depends neither on the source fields $\eta^{+}$and $\eta$ nor on the integration variables $\phi^{+}$and $\phi$ and, hence, does not contribute to the derivatives of $\log Z$ with respect to the source fields (see the discussion in Sec. III). Furthermore, we represent 
$\widetilde{M}\left[\phi^{+}, \phi\right]=\mathbb{1}+\widetilde{\mathcal{M}}\left[\phi^{+}, \phi\right]$ where

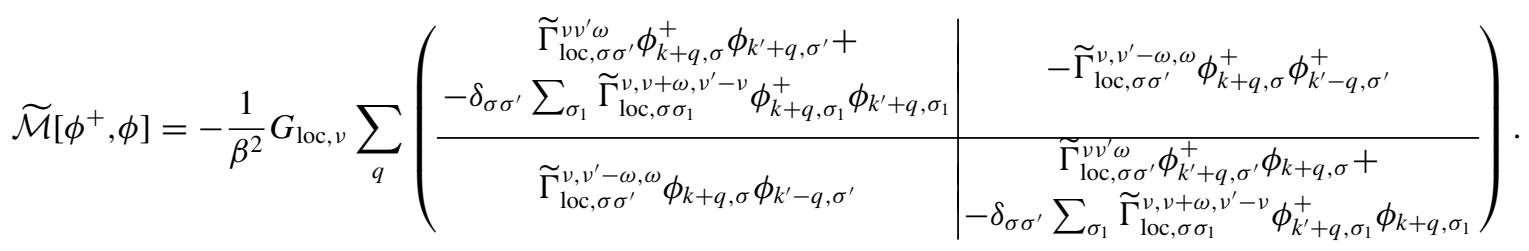

The inverse (note that we are dealing with Grassmann integrals) ${ }^{37}$ of $J\left[\phi^{+}, \phi\right]$ is now given by

$$
J^{-1}\left[\phi^{+}, \phi\right]=\operatorname{det} \tilde{M}\left[\phi^{+}, \phi\right] .
$$

Here, det denotes the determinant with respect to the $k$ and $\sigma$ indices. In order to include $J$ in the effective action, we transfer it to the exponent by taking its logarithm and make use of the general identity $\log \operatorname{det} A=\operatorname{Tr} \log A$. Hence, we finally arrive at

$$
\log J\left[\phi^{+}, \phi\right]=-\operatorname{Tr} \log \tilde{M}\left[\phi^{+}, \phi\right]=-\operatorname{Tr} \log \left(\mathbb{1}+\widetilde{\mathcal{M}}\left[\phi^{+}, \phi\right]\right) .
$$

where $\operatorname{Tr}$ denotes the trace with respect to the $k$ and $\sigma$ indices. Performing a Taylor expansion of the logarithm in the last term, we obtain an expansion of the Jacobian in $\phi^{+}, \phi$ fields.

In the first (quadratic) order in fermionic fields we obtain the term with the structure $G_{\mathrm{loc}} \Gamma_{\mathrm{loc}} \phi^{+} \phi$. Hence, it corresponds to the first diagram in Fig. 3(b) and cancels the corresponding ones which are generated by the perturbation expansion of the 1PI functional Eq. (20). The terms of the second (quartic) order can be schematically written as $\Gamma_{\mathrm{loc}} G_{\mathrm{loc}}^{2} \Gamma_{\mathrm{loc}}\left(\phi^{+} \phi\right)\left(\phi^{+} \phi\right)$ and correspond to the second diagram in Fig. 3(b). Let us also note that higher-order contributions in $\phi$, i.e., $O\left(\left(\phi^{+} \phi\right)^{3}\right)$, generate terms that cancel the nonlocal corrections to the self-energy stemming from the three- (and more)-particle local 1PI vertices that are already taken into account at the two-particle vertex level via combination of the elements of diagram technique of Fig. 3. In this way any possible double counting is avoided in the 1PI approach. For a more detailed discussion of this issue we refer to Ref. 31 .

\section{Transformation of integral variables and decoupling of the three-particle term}

In this section we decouple the term in the second line of Eq. (20), which contains a three-particle interaction, as discussed below Eq. (21). For this purpose we consider the following Hubbard-Stratonovich transformations:

$$
\begin{aligned}
& \exp \left\{\beta\left(\frac{\delta \Gamma_{\mathrm{DMFT}}\left[\phi^{+}, \phi\right]}{\delta \phi_{k \sigma}}+\eta_{k \sigma}^{+}\right)\left[\zeta_{v}^{-1}-G_{0 k}^{-1}\right]^{-1}\left(-\frac{\delta \Gamma_{\mathrm{DMFT}}\left[\phi^{+}, \phi\right]}{\delta \phi_{k \sigma}^{+}}+\eta_{k \sigma}\right)\right\} \\
& =\int d \psi_{k \sigma}^{+} d \psi_{k \sigma} \exp \left\{-\frac{1}{\beta}\left[\zeta_{v}^{-1}-G_{0 k}^{-1}\right] \psi_{k \sigma}^{+} \psi_{k \sigma}\right\} \exp \left\{\left[\left(\frac{\delta \Gamma_{\mathrm{DMFT}}\left[\phi^{+}, \phi\right]}{\delta \phi_{k \sigma}}+\eta_{k \sigma}^{+}\right) \psi_{k \sigma}\right.\right. \\
& \left.\left.\quad+\psi_{k \sigma}^{+}\left(-\frac{\delta \Gamma_{\mathrm{DMFT}}\left[\phi^{+}, \phi\right]}{\delta \phi_{k \sigma}^{+}}+\eta_{k \sigma}\right)\right]\right\},
\end{aligned}
$$

where we neglected the prefactor $\beta\left[\zeta_{v}^{-1}-G_{0 k}^{-1}\right]^{-1}$ in front of the functional integral in this equation, since it drops out in the calculation of the Green's function. In the next step we insert Eq. (A9) into Eq. (20) and then perform the following shift of integration variables:

$$
\psi_{k \sigma}^{+} \rightarrow \psi_{k \sigma}^{+}+\phi_{k \sigma}^{+}, \quad \psi_{k \sigma} \rightarrow \psi_{k \sigma}+\phi_{k \sigma} .
$$

One observes that the terms $\left(\delta \Gamma_{\mathrm{DMFT}} / \delta \phi\right) \phi$ and $\phi^{+}\left(\delta \Gamma_{\mathrm{DMFT}} / \delta \phi^{+}\right)$in Eq. (20) are canceled by the corresponding ones from Eq. (A9). Hence, one arrives at the following expression for the generating functional $Z\left[\eta^{+}, \eta\right]$ :

$$
\begin{aligned}
Z\left[\eta^{+}, \eta\right]= & \int D\left[\phi^{+}, \phi\right] \exp \left\{-\frac{1}{\beta} \sum_{k, \sigma}\left[\zeta_{v}^{-1}-G_{0 k}^{-1}\right]\left(\psi_{k \sigma}^{+}+\phi_{k \sigma}^{+}\right)\left(\psi_{k \sigma}+\phi_{k \sigma}\right)+\frac{\delta \Gamma_{\mathrm{DMFT}}\left[\phi^{+}, \phi\right]}{\delta \phi_{k \sigma}} \psi_{k \sigma}\right. \\
& \left.-\psi_{k \sigma}^{+} \frac{\delta \Gamma_{\mathrm{DMFT}}\left[\phi^{+}, \phi\right]}{\delta \phi_{k \sigma}^{+}}-\Gamma_{\mathrm{DMFT}}\left[\phi^{+}, \phi\right]+\eta_{k \sigma}^{+}\left(\psi_{k \sigma}+\phi_{k \sigma}\right)+\left(\psi_{k \sigma}^{+}+\phi_{k \sigma}^{+}\right) \eta_{k \sigma}\right\} J\left[\phi^{+}, \phi\right] .
\end{aligned}
$$

Inserting now the explicit expressions for $\Gamma_{\mathrm{DMFT}}\left[\phi^{+}, \phi\right]$ from Eq. (21) and $\left(\delta \Gamma_{\mathrm{DMFT}} / \delta \phi^{(+)}\right)$from Eq. (A3) into Eq. (A11) one arrives at the final expression for the generating functional $Z\left[\eta^{+}, \eta\right]$ in the 1PI representation as given in Eq. (22) [consider that $\left.-\zeta_{v}^{-1}+G_{0 k}^{-1}+G_{\mathrm{loc}, v}^{-1}=G_{k}^{-1}\right]$. 
${ }^{1}$ W. Metzner and D. Vollhardt, Phys. Rev. Lett. 62, 324 (1989);

A. Georges and G. Kotliar, Phys. Rev. B 45, 6479 (1992).

${ }^{2}$ A. Georges, G. Kotliar, W. Krauth, and M. Rozenberg, Rev. Mod. Phys. 68, 13 (1996); G. Kotliar and D. Vollhardt, Phys. Today 57, 53 (2004).

${ }^{3}$ N. F. Mott, Rev. Mod. Phys. 40, 677 (1968); Metal-Insulator Transitions (Taylor \& Francis, London, 1990); F. Gebhard, The Mott Metal-Insulator Transition (Springer, Berlin, 1997).

${ }^{4}$ M. H. Hettler, A. N. Tahvildar-Zadeh, M. Jarrell, T. Pruschke, and H. R. Krishnamurthy, Phys. Rev. B 58, 7475 (1998).

${ }^{5}$ A. I. Lichtenstein and M. I. Katsnelson, Phys. Rev. B 62, R9283 (2000).

${ }^{6}$ G. Kotliar, S. Y. Savrasov, G. Palsson, and G. Biroli, Phys. Rev. Lett. 87, 186401 (2001).

${ }^{7}$ T. A. Maier, M. Jarrell, T. Pruschke, and M. H. Hettler, Rev. Mod. Phys. 77, 1027 (2005).

${ }^{8}$ A. Schiller and K. Ingersent, Phys. Rev. Lett. 75, 113 (1995).

${ }^{9}$ A. Toschi, A. A. Katanin, and K. Held, Phys. Rev. B 75, 045118 (2007); K. Held, A. A. Katanin, and A. Toschi, Prog. Theor. Phys. Suppl. 176, 117 (2008); A. Valli, G. Sangiovanni, O. Gunnarsson, A. Toschi, and K. Held, Phys. Rev. Lett. 104, 246402 (2010); A. Toschi, G. Rohringer, A. Katanin, and K. Held, Ann. Phys. (N.Y.) 523, 698 (2011).

${ }^{10}$ Similar, albeit less elaborated, ideas were also independently developed by H. Kusunose, J. Phys. Soc. Jpn. 75, 054713 (2006). Moreover, a related method, but with a cluster instead of a single site as a starting point, was developed by C. Slezak, M. Jarrell, Th. Maier, and J. Deisz, J. Phys.: Condens. Matter 21, 435604 (2009).

${ }^{11}$ A. N. Rubtsov, M. I. Katsnelson, and A. I. Lichtenstein, Phys. Rev. B 77, 033101 (2008); S. Brener, H. Hafermann, A. N. Rubtsov, M. I. Katsnelson, and A. I. Lichtenstein, ibid. 77, 195105 (2008).

${ }^{12}$ H. Hafermann, G. Li, A. N. Rubtsov, M. I. Katsnelson, A. I. Lichtenstein, and H. Monien, Phys. Rev. Lett. 102, 206401 (2009).

${ }^{13}$ A systematic description of the diagrammatics and of the corresponding numerical results for the one- and two-particle irreducible local vertex functions can be found in Refs. 32,33.

${ }^{14}$ We note, however, that in the diagrammatic approach to the Anderson localization of Refs. 15,16 , by taking the infinite dimensional limit corresponding to DMFT the exact solution reduces to the coherent potential approximation (CPA). This simplification, of course, does not apply to the case of the Hubbard model problems considered in the present work.

${ }^{15}$ V. Janiŝ, Phys. Rev. B 64, 115115 (2001).

${ }^{16}$ V. Janiŝ and J. Kolorenĉ, Phys. Rev. B 71, 033103 (2005); 71, 245106 (2005).

${ }^{17}$ A. A. Katanin, A. Toschi, and K. Held, Phys. Rev. B 80, 075104 (2009).

${ }^{18}$ S.-X. Yang, H. Fotso, H. Hafermann, K.-M. Tam, J. Moreno, T. Pruschke, and M. Jarrell, Phys. Rev. B 84, 155106 (2011).

${ }^{19}$ T. A. Maier, M. S. Jarrell, and D. J. Scalapino, Phys. Rev. Lett. 96, 047005 (2006).

${ }^{20}$ C. De Dominicis, J. Math. Phys. 3, 983 (1962); C. De Dominicis and P. C. Martin, ibid. 5, 14 (1964); A. D. Jackson, A. Lande, and R. A. Smith, Phys. Rep. 86, 55 (1982); A. Lande and R. A. Smith, Phys. Rev. A 45, 913 (1992); N. E. Bickers and S. R.
White, Phys. Rev. B 43, 8044 (1991); N. E. Bickers, Int. J. Mod. Phys. B 5, 253 (1991); V. Janiŝ, Phys. Rev. B 60, 11345 (1999).

${ }^{21}$ S. X. Yang, H. Fotso, J. Liu, T. A. Maier, K. Tomko, E. F. D'Azevedo, R. T. Scalettar, T. Pruschke, and M. Jarrell, Phys. Rev. E 80, 046706 (2009); Ka-Ming Tam, H. Fotso, S.-X. Yang, Tae-Woo Lee, J. Moreno, J. Ramanujam, and M. Jarrell, ibid. 87, 013311 (2013).

${ }^{22}$ G. Rohringer, A. Toschi, A. A. Katanin, and K. Held, Phys. Rev. Lett. 107, 256402 (2011).

${ }^{23}$ A. Katanin, J. Phys. A: Math. Theor. 46, 045002 (2013).

${ }^{24}$ J. Bickers, in Theoretical Methods for Strongly Correlated Electrons, edited by D. Senechal, A. M. Tremblay, and C. Bourbonnais (Springer, New York, 2004), ch. 6; D. B. McWhan, A. Menth, J. P. Remeika, W. F. Brinkman, and T. M. Rice, Phys. Rev. B 7, 1920 (1973).

${ }^{25}$ The equivalence of Eq. (16) and (15) just follows from integrating out the $\tilde{c}$ fields.

${ }^{26}$ Replacing $G_{0}$ by the (interacting) DMFT Green's function $G$ and substituting correspondingly the Weiss-field (i.e., the noninteracting local Green's function) $\zeta$ by its interacting counterpart $G_{\text {loc }}$ leads to exactly the same decoupling scheme as used in DF. ${ }^{11}$ This can be easily seen from the identity $\zeta_{v}^{-1}-G_{0 k}^{-1}=G_{\mathrm{loc}, v}^{-1}-G_{k}^{-1}$. However, we think that the way of splitting local and nonlocal degrees of freedom as performed in Eq. (16) is physically more transparent.

${ }^{27}$ A. N. Rubtsov, M. I. Katsnelson, A. I. Lichtenstein, and A. Georges, Phys. Rev. B 79, 045133 (2009).

${ }^{28}$ We recall that in DF self-consistency can be performed at twodifferent levels: (i) by reinserting the Green's functions of the dual electrons in the self-energy diagrams of the dual electrons (inner self-consistency) and (ii) by readapting the AIM to take into account that the local physics has also changed due to the nonlocal DF corrections (outer self-consistency).

${ }^{29} \mathrm{In}$ DГ А, as well in 1PI, one can correct for the non-self-consistency effects appearing for the two-particle quantities in one-shot calculations for the ladder diagrams, by correcting the two-particle susceptibilities within the Moriya schemes, as explained in Ref. 17.

${ }^{30}$ Gang Li, Hunpyo Lee, and Hartmut Monien, Phys. Rev. B 78, 195105 (2008).

${ }^{31} \mathrm{G}$. Rohringer (unpublished).

${ }^{32}$ G. Rohringer, A. Valli, and A. Toschi, Phys. Rev. B 86, 125114 (2012).

${ }^{33}$ T. Schäfer, G. Rohringer, O. Gunnarsson, S. Ciuchi, G. Sangiovanni, and A. Toschi, Phys. Rev. Lett. 110, 246405 (2013).

${ }^{34}$ P. R. C. Kent, M. Jarrell, T. A. Maier, and T. Pruschke, Phys. Rev. B 72, 060411 (2005).

${ }^{35}$ R. Staudt, M. Dzierzawa, and A. Muramatsu, Eur. Phys. J. B 17, 411 (2000); S. Fuchs, E. Gull, L. Pollet, E. Burovski, E. Kozik, T. Pruschke, and M. Troyer, Phys. Rev. Lett. 106, 030401 (2011).

${ }^{36}$ For the 1PI approach the self-consistency could be alternatively achieved as in DF, i.e., by redefining the local problem $\zeta\left(i \omega_{n}\right)$, so to minimize the corrections with respect to the new local AIM.

${ }^{37}$ J. W. Negele and H. Orland, Quantum Many-Particle Systems (Westview Press, Boulder, 1998)

${ }^{38}$ D. Rost, E. V. Gorelik, F. Assaad, and N. Blümer, Phys. Rev. B 86, 155109 (2012). 
${ }^{39}$ H. Park, K. Haule, and G. Kotliar, Phys. Rev. Lett. 101, 186403 (2008).

${ }^{40}$ K. Borejsza and N. Dupuis, Europhys. Lett. 63, 722 (2003); Phys. Rev. B 69, 085119 (2004).

${ }^{41}$ For a DMFT-based analysis of the antiferromagnetic crossover for the particle-hole symmetric model, see G. Sangiovanni et al.,
Phys. Rev. B 73, 205121 (2006); C. Taranto, G. Sangiovanni, K. Held, M. Capone, A. Georges, and A. Toschi, ibid. 85, 085124 (2012).

${ }^{42}$ W. Metzner, M. Salmhofer, C. Honerkamp, V. Meden, and K. Schoenhammer, Rev. Mod. Phys. 84, 299 (2012). 